\title{
Stakeholder's profitability of carrier-led consolidation strategies in urban goods distribution
}

\begin{abstract}
This paper presents the necessary conditions to ensure a minimal profitability of carrier-led consolidation strategies in urban distribution. These conditions are shown by compact formulas obtained by continuous approximations representing the cost of the stakeholders involved: society, regular carriers, consolidation facility operator and environment. The domain of the retailer density variable that always produces negative effects on each stakeholder has been identified. The envelope of this domain does not depend on vehicle costs and other site-related parameters. On the other hand, there is a critical density of receivers that makes the carrier cost savings higher than the $C F$ operator costs.
\end{abstract}

Keywords: city logistics, consolidation strategies, urban consolidation centers, last-mile network, freight modeling

\section{Introduction}

The predominance of population concentration in urban areas was achieved for first time in the human history in 2008 (UNFPA, 2007). From this time on, world population is expected to continue concentrating in cities or metropolitan areas due to incremental access to economic opportunities and services (UNFPA, 2012). Cities must provide efficient networks of these basic services to meet the near future citizens' needs at a reasonable cost. This is the case of the urban goods distribution. In order to improve its efficiency and to reduce externalities, local governments have been implementing heterogeneous innovative measures, through urban mobility plans or using national or supranational funds. City managers have done a great effort in developing detailed ex-post evaluation methodologies to comprehend the results, derive lessons to other implementations and benchmark the best practices in city logistics. Unfortunately, ex-ante estimations of these urban goods measures are seldom provided. The cause-effect relationships are not studied and there is no estimation of the required conditions to guarantee the achievement of the objectives.

Consolidation strategies are one set of measures that can improve the efficiency and reduce the externalities caused by freight vehicles in urban areas (Browne et al, 2005). The basic idea is to consolidate goods in new or existing facilities before the distribution to the final customers is done. This way, the number of freight vehicles and their mileage within an urban area are reduced by grouping the shipments of different delivering routes into a single vehicle covering the destination area. However, there is a major challenge for the success of consolidation strategies: which stakeholder leads the consolidation and how the new distribution costs of the consolidation facility are compensated by the stakeholders that experience a cost reduction.

In Holguín-Veras and Sánchez-Díaz (2016), two different kinds of organizational implementation of consolidation strategies are identified. On one hand, receiver-led consolidation strategies, where the major decision role relies on receivers. A common example is when the manager of a great pole freight attractor (commercial malls, hospitals, huge office buildings) reorganizes the upstream supply chains, reducing the number of deliveries in this facility. This measure may reduce the number of in-bound routes but it does not need any collaboration among carriers.

On the other hand, carrier-led consolidation strategies, where receivers are physically scattered in the urban area and they are not organized under a common framework or legal body. In that case, multiple carriers use a consolidation facility to deliver goods in the distribution area. Each carrier is responsible for performing multiple deliveries to a subset of these receivers (Nathanail et al, 2012). Indeed, these strategies cause significant changes in the inbound supply chains at the facility, as well as in the outbound local distribution routes, from the facility to the final customers. Interurban vehicles just need to stop once at this facility to deliver all goods to receivers located in this area. In this facility, the shipments are consolidated and a common fleet distributes them to the final destinations. Consolidation strategies imply some level of collaboration among carriers since their parcels are combined in the common fleet for lastmile delivery or, at least, are handled in a shared space in the consolidation facility. However, the natural competition of carriers makes this kind of consolidation strategies more complex than the receiver-led. The main objective of carrier-led consolidation measure is to increase the load factor in the last mile 
distribution, to reduce the number of gross vehicles in the area and to reduce the environmental impacts of the freight distribution while maintaining the level of service.

A well-known example of these carrier-led consolidation strategies is the controversial Urban Consolidation Center (UCC) concept or also named Urban Logistic Spaces (ULS). They are medium logistic facilities located in the neighborhood of the distribution area in which deliveries are consolidated. In Browne et al (2005) a deep analysis of the potentialities and major weaknesses of UCC is presented. In spite of the promising expected results, one of the major challenges is the necessary funding to afford the set-up facility cost. Moreover, this measure implies new handling costs as well as time penalty for all shipments to be distributed through UCCs. The economic impacts on multiple stakeholders involved in several UCC trials have not been deeply estimated. It has resulted in unfeasible business models. In fact, more than 100 implementation of this kind all over the world were reported, but just 12 are still in service (Allen et al., 2012). Several contributions present a quantified assessment of the impacts of UCC in specific implementation in Europe, North American and Japan cities (Paddeu et al, 2014, Köhler, 2001, Kawamura and Lu, 2008, Chen et al, 2012, Browne et al, 2007, and Gonzalez-Feliu and Morana, 2011). These results apply to each case study and it is difficult to extrapolate the achievements to other physical contexts.

Alternatively, the concept of Urban Stage Areas, uSA, (referred as Espace Logistique de Proximité in France) has succeeded in the recent years as a consolidation strategy (Holguín-Veras et al. 2008). It overcomes the funding problem of building a new unaffordable consolidation facility. A container or other small non-expensive storage structure is installed in an existing facility inside a city center (for example, a parking garage). Vehicles drop the products to be delivered in the adjacent area of this facility and, in a second stage, other environmentally-friendly vehicles from the same company deliver them to the final retailers. Even, this last mile distribution can be made by foot. In Janjevic et al (2013), the special features of $U L S$ are analyzed.

The estimation of the logistic cost savings from carrier-led consolidation strategies is addressed in several contributions based on continuous approximations of the network distribution variables (Daganzo 1988a, b, and Campbell, 1990). Saberi and Verbas (2012) presented a similar approach aimed at minimizing the emissions of the freight transportation vehicles due to carrier-led consolidation policies. Roca-Riu et al (2012) and Roca-Riu et al (2016) analyzed the effect of equal and non-equal market share carriers demand in the whole transportation network. The total transportation cost variation at local and line-haul network are estimated. These contributions provide a useful tool to approximate the total cost of both systems and therefore the operational savings. More recently, Janjevic and Ndiaye (2016) define the generalized cost of regular carriers when they route their parcels through UCC. This generalized cost is obtained as an analytical estimation of the time and distance- related cost as well as the service fare. Unfortunately, there is not any analysis of the consolidation effect on the profitability of each stakeholder involved and the necessary cash flows among them. In this paper, we would like to study how the cost incurred by each agent (mainly carriers and consolidation facility operator) changes due to the consolidation strategy and what novel cash flows among stakeholders ensure the profitability for each participating agent. Zhou et al. (2011) analyzed the cost savings and the optimal price setting of companies. Although the study is developed for the long-haul network, they define an economic model where the product substitutability among carriers is addressed, considering price setting and discount offer. In the urban context, Krajewska and Kopfer (2006) and Krajewska et al. (2008) addressed the cost allocation problem among companies. This problem studies the distribution of new costs and its benefits due to the collaborative process among participants. The methodological framework of this approach considers both combinatorial auctions and operational research game theory. Unfortunately, these latter contributions do not provide insights about how the distribution network should be designed to exploit the economies of scale of consolidation facilities at a specific site.

The aim of this paper is to define the necessary conditions to ensure a minimum profitability of the carrier-led consolidation strategies for carriers, the consolidation facility operator, environment and society. These conditions are determined using a set of compact formulas involving the logistic cost estimation and the cash flow among stakeholders. Cost estimations are compared between the regular service and the service with consolidation facilities. Formulas depend on the key characteristics of the urban site and distribution network: demand and size of parcels, size and type of fleet, local and line-haul speeds, unit cost parameters of the resources involved (time, distance, infrastructure, ...) and the fares to use the consolidation facility. In Section 2, there is a comprehensive analysis of the economic and organizational relationships among the key stakeholders involved in consolidation strategies. Section 3 presents an analytic model to estimate the cost variation incurred by stakeholders due to the participation in a consolidation facility. Section 4 shows the necessary conditions to implement consolidation 
strategies, ensuring a positive profitability for all stakeholders. Section 5 analyzes a generic case study. Finally, the major modeling and applicability conclusions are drawn in Section 6.

\section{Stakeholders and their relationships}

The promotion of a consolidation strategy represents a new scheme in the logistics system of a city, changing the roles and the logistic cost allocation among stakeholders involved. In this paper, we will focus on carrier-led consolidation strategies and two typologies of consolidation facilities will be studied: Urban Consolidation Centers (UCC) and Urban Stage Areas (uSA). From now on, we will use indistinctively the term Consolidation Facility $(C F)$.

According to Holguin-Veras and Sánchez-Diaz (2016), the main stakeholders that determine the economic characteristics of urban distribution service are the suppliers and receivers. They represent the producer and the consumer of the supply chain under analysis. The economic relations between production and consumption are the key to understand the behavior of carriers and how distribution services are organized. However, carrier-led consolidation strategies which use a consolidation facility imply a new stakeholder in the supply chain: the operator of the consolidation facility (CF operator).

In Figure 1, the basic relationships among stakeholders are depicted, identifying the economic, physical and regulatory layers placing the stakeholders and defining their relationships. The overall list of variables used throughout the paper are listed in Appendix 1. Subscripts $C$ and $C F$ refer to carrier and $C F$ operator respectively. Basically, in the economic layer, each economic relationship $k$ between supplier and receiver determines the amount of products to be delivered $y_{k}$, the frequency of shipments, the pickup/delivery time, and the time horizon $\left(H_{k}\right)$ that carrier $i$ must fulfill. Reciprocally, carrier $i$ defines the transportation fare $\theta_{C}(k)$ to route this parcel through the physical network and adapt their fleet $\left(V_{C}\right)$, schedule and routes $\left(R_{C}\right)$ to fulfill the requirements of the former stakeholders in order to visit $N_{i}$ receiver locations. On the regulation layer, local governments foster carrier-led consolidation measures due to the improvement of the quality of life in the area (less congestion, less emissions). These sets of compulsory measures $\left(\chi_{L}\right)$ are usually intended for carriers, constraining $V_{C}, R_{C}$.

Hence, the agents that foresee a completely different economic balance due to consolidation strategies are those that operate the physical distribution network, i.e. carriers and CF operator. When they move a fraction $\mu$ of the total receivers $N_{i}$ from a traditional network to the new consolidated supply chain, they only operate the line-haul service from distribution centers to the urban $C F$. Then, the $C F$ operator handles the transfer operations of goods from inbound $C F$ operator routes (interurban) and performs the local routes in the urban area to deliver goods to the final receivers. This stakeholder incurs the investment cost of the facility construction and the operational cost of the last-mile distribution. The lastmile distribution routes $R_{C F}$ and fleet $V_{C F}$ from this consolidation facility must fulfill the requirements of receivers as well as the specific regulations imposed by local authorities $\left(\chi_{\mathrm{CF}}\right)$.

However, the key question in this analysis is how the total cost sustained by the CF operator is balanced by incomes or compensations in order to ensure a positive profitability for all stakeholders. We consider that the $C F$ operator charges a fare $\theta_{C F}(k)$ to the parcel corresponding to the $k$-th supplier-receiver relationship served through the consolidation facility. This fare could be complemented by a subsidy $\left(S_{C F}\right)$ from the local authority (Kin et al, 2016, Lebeau et al, 2015) or other incomes to ensure a positive profit for the $C F$ operator (i.e., advertisement, storage, ...).

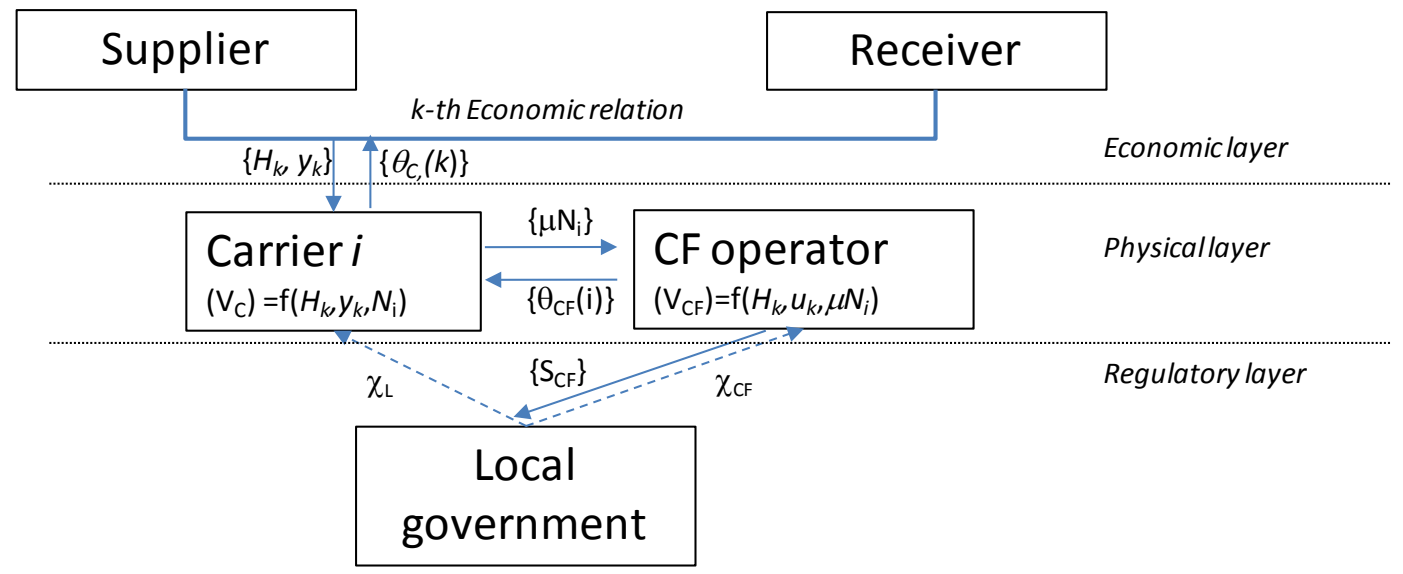

Figure 1: Economic and physical relationship among stakeholders 


\section{Consolidation modeling approach}

In this section, the cost variation due to the consolidation facilities is estimated. Formulas are developed to assess the temporal and distance costs difference between regular distribution and the distribution through a Consolidation Facility (CF). We refer as a regular distribution the supply of goods from distribution centers directly to the final receivers. The basic equations estimating the logistic cost of regular distribution are firstly developed for a single carrier. Later, we introduce the case where different market share carriers operate in the same area of the city in the regular distribution. Finally, we analyze the effects of the $C F$ service on the overall costs of carriers under competition.

\subsection{Single carrier}

We consider that one carrier has to visit $N$ receivers located in a rectangular zone of a city of area $A=l_{x}$. $l_{y}$, where $l_{x}, l_{y}$ correspond to each rectangle side length, respectively. Let $\delta$ be the spatial density of receivers in the area of study. The key assumptions of the methodological approach are:

- Receivers are uniformly distributed over the region of service $l_{x} l_{y}$, so that $\delta=N / A$ is considered uniform in all area of service. The delivery area is assumed to be a square, circle, or a shape that is not very elongated.

- The maximal allowable parcel volume to be served in regular distribution is $y_{\max }$. Parcels greater than this threshold are transported through an alternative supply chain devoted to special shipments. Let $y_{i}$ be the volume of the parcel requested by each receiver $i(i=1, \ldots, N)$ and $F(y)$ the cumulative distribution function of parcels whose volume is less or equal to $y_{\max }$ in the total area of service, satisfying $F(y=0)=0$ and $F\left(y=y_{\max }\right)=1$.

- Each receiver has the same probability of requesting a shipment of volume $y\left(0 \leq y \leq y_{\max }\right)$. Moreover, the expected value of the parcel volume served by carrier in the region is estimated by $E(y)=\int_{0}^{y_{\max }} y f(y) d y$, where $f(y)$ is the corresponding probability density function of parcel volume. We generally assume that $F(y)$ and $f(y)$ are deterministic functions that can be directly obtained from the data of those parcels served by the carrier.

- Carriers will use a homogeneous fleet of volume capacity C. As a difference from Daganzo (1984a, 1984b, 2005), the vehicle capacity $C$ is defined as the total volume of parcels that one vehicle can carry whereas $C / E(y)$ is the expected maximal number of receivers that one tour is able to visit.

- Vehicle tours are designed within the time horizon $H$. It captures the available time period in which receivers admit the deliveries during the day.

- Vehicles depart from a depot (distribution center) located at distance $\rho$ from the center of the service area. As a difference from Daganzo (1984b) and Robusté et al (1990), we assumed that the depot is deployed outside the city boundaries $(\rho>\sqrt{A})$; therefore we cannot consider it is located near the center of the region to be served.

- Given the density of streets in urban areas, we use the $\mathrm{L}_{1}$ metric to determine the distance evaluation in the service area for the simplicity. We assume that vehicles may detour an extra distance $\beta$ in each tour due to the single direction of streets or access regulations. This term could be important in historical centers with a compact road street network and one-way streets. In Holroyd (1965), there are several routing factor equivalences between distance estimations provided for different street network shapes and metrics.

The design of carrier routes in the regular distribution is aimed at minimizing the generalized cost of the trip. The region is firstly partitioned in clusters of approximately shipment volume $S$, each of one presenting $S / E(y)$ receivers as an average. Therefore, decision variable $S$ is limited by temporal and capacity constraints (parameters $C$ and $H$ ). We will refer as line-haul distance to the total distance run from the distribution center to the central point of each urban distribution area by the whole fleet. On the other side, local distance is the distance covered during the delivery of parcels within the cluster of receivers. Newell and Daganzo (1986) proposed a simple (non-optimal) strategy for visiting the points contained in zones elongated towards the distribution center. Zones are rectangles with sides $2 w$ and $P$, which constitute a partition of the whole delivery region. If vehicles visit receivers at full load and there is no time limitation, the number of receivers in the rectangle should be $C / E(y)$, which should be equivalent to $2 w P \delta$, expressed in terms of density. However, we might be also interested in reducing the average number of points to $S / E(y)$ to satisfy the time horizon $H$. 


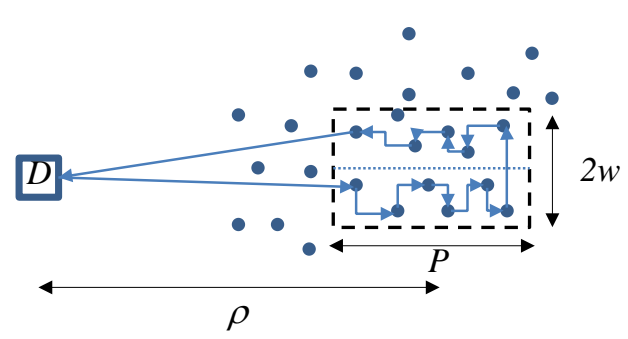

Figure 2. Scheme of the receiver partition in the regular distribution network of one carrier.

From the contributions of Daganzo (1984a,b) and adding the line-haul, local distances and detour distance, we obtain an approximation of the total distance traveled per receiver, $d$ (Equation 1 ). In the case of Figure 2, parameter $\beta$ could be approximated by the available street spacing in the transversal direction between two adjacent bands.

$$
d=\frac{w}{3}+\frac{1}{\delta w}+\frac{2 \rho}{S / E(y)}+\frac{\beta}{S / E(y)}
$$

Using $v_{L}, v_{L H}$ and $\tau$ as urban speed inside the local service area, interurban speed from the distribution center to the service area, and the unloading/loading time per receiver respectively, the total time per receiver $(t)$ is estimated in Equation (2):

$$
t=\frac{2 \rho E(y)}{S v_{L H}}+\frac{\beta E(y)}{S v_{L}}+\frac{1}{w \delta v_{L}}+\frac{w}{3 v_{L}}+\tau
$$

Therefore, the carrier is aimed at minimizing transportation cost while the service is maintained to all receivers. We consider $c_{d}$ and $c_{t}$ as the distance and time-related unit costs respectively. If we let $D_{L H}, D_{L}$ and $T$ be the total line-haul distance, total local distance and total travel time, Equation (3) determines the objective function to be minimized for a single carrier.

$$
\min Z(w, S)=\min \left[c_{d}\left(D_{L}+D_{L H}\right)+c_{t} T\right]
$$

The decision variables are: the dimension of the vehicle zone delivery partition $(w)$ and the vehicle volume load $(S)$. The expressions of the optimal distances and the travel time that one carrier need to travel to visit $N$ receivers are provided in equations (4)-(6) respectively. In Appendix 2, the methodological approach to obtain the former formulas is presented. The term $[N / \psi]^{+}$captures the integer number of tours needed to serve all receivers, where the mathematical operator $[x]^{+}$gives the smallest integer greater than or equal to $x$.

$D_{L}=\frac{2}{\sqrt{3}}(A N)^{1 / 2}+\beta\left[\frac{N}{\psi}\right]^{+}$

$D_{L H}=2 \rho\left[\frac{N}{\psi}\right]^{+}$

$T=\left(D_{L} / v_{L}+D_{L H} / v_{L H}\right)+\tau N$

Daganzo (1984a) and Robuste et al (1990) demonstrated that the former formulas are good distance approximations when $N>>C$. If $C=N$, the local areas can not be built towards the depot since they are not long and narrow. In these cases, one vehicle is able to visit all points, and, as in the former references, the depot is located at the center of service area, the line haul distance estimation (term $2 \rho$ ) is not correct. In fact, the former contributions justify that Equations (4) - (5) are only valid when $7<C<1.5(N / C)$. When the second inequality is not satisfied, the line haul distance of Equation (5) should be replaced by $D_{L H}=\left(0.4 N / C^{2}\right)(A N)^{1 / 2}$ for circular zones and depot located at the center. However, in our paper this latter assumption is not valid. We consider that the depot will be never located at the center of the city area since it is deployed in the surroundings of the city far away from the delivering zones $(\rho>\sqrt{A})$. Therefore, for $N \approx C$, the line-haul distance component cannot be neglected and its numerical value will be, at least similar or, higher than local distance. In those cases, the validity of the line-haul distance estimation is justified. The other term under analysis, the local distance estimation $(\sqrt{A N})$, is a good approximation in compact areas even if $N$ is a low number. Daganzo (1984b) justified that, when $N=2$ receivers, the deviation between the modeled distance and the real one is within $0.02 \sqrt{A}$ in rounded or squared zones. 


\subsection{Several carriers}

The formulas derived above for a single carrier delivering parcels to a set of $N$ receivers are now used to compare two alternative logistic scenarios: $(A)$ independent carriers performing last-mile delivery in a regular distribution, and $(B)$ a last-mile delivery system with collaboration among carriers and freight consolidation through a Consolidation Facility (UCC or $u S A)$.

The following assumptions and the ones presented in Section 3.1 apply to the methodological approach to be developed:

- Now, we consider that the distribution service is performed by $M$ different carriers. We assume that each carrier $i=1, \ldots, M$ gives service to a subset of $N_{i}$ customers, with $N=\sum_{i=1,, M} N_{i}$. Indeed, the variable $\phi_{i}=N_{i} / N$ denotes the market share of each carrier $i(i=1, . ., M)$ considering the total distribution of parcels in the area of service. The market share can be different among carriers. Therefore, the customer density of each company is denoted by $\delta_{i}=N_{i} / A, i=1, . ., M$. Note that $\delta_{i}$ is smaller than the overall demand density in the region $\delta=N / A$. The parcel volume distribution $F(y)$ is considered to be the same for all carriers involved.

- The consolidation facility is located inside the delivering area, at a distance $\varphi=\varphi_{x}+\varphi_{y}$ from the center of the service region $\left(0 \leq \varphi_{x} \leq l_{x} / 2,0 \leq \varphi_{y} \leq l_{y} / 2\right)$.

- Carriers still use vehicles with the same capacity in the line-haul distribution (from distribution center to the urban consolidation facility). However, the $C F$ operator may use bigger or smaller vehicles in the last mile distribution network with regard to the carrier's fleet. Indeed, we consider the capacity of those vehicles used by $C F$ operator in the last mile network to be $C_{C F}=$ $k_{C} C$, where $k_{c} \in \mathbb{R}^{+}$. Although the parcel consolidation at urban facilities would allow the usage of bigger vehicles $\left(k_{C} \geq 1\right)$ in the local distribution, the number of routes can be constrained by the available time horizon rather than capacity restriction. In fact, $C F$ operator may choose vehicles of smaller capacity $\left(k_{C} \leq 1\right)$ that will fit better the vehicle size regulations of the city and the physical layout of existing streets. These vehicles may show lesser detour distance in each tour.

- The maximal parcel volume of a receiver $u_{\max }\left(u_{\max } \leq y_{\max }\right)$ that is accepted to be delivered with the vehicles operated by $C F$ operator is assumed to be $u_{\max }\left(u_{\max } \leq y_{\max }\right)$. We suppose that all parcels with $y \leq u_{\max }$ will be routed through the consolidation facility and transshipped to the vehicles of $C_{C F}$ capacity (strategy B). Now, we refer the volume of each parcel $k$ to be routed through the consolidation facility as $u_{k}\left(0 \leq u_{k} \leq u_{\max }\right)$. Therefore, this $u_{\max }$ constitutes a decision variable that plays a key role in the $C F$ operator profitability. The definition of $u_{\text {max }}$ should be done by $C F$ operator jointly with the selection of the nominal capacity of vehicles $C_{C F}$, taking into account the distribution of parcel volumes $F(y)$. Therefore, the total number of deliveries to be captured by the consolidation service from a single carrier $i(i=1, . ., M)$ will be estimated by $N_{i} F\left(u_{\text {max }}\right)$. Under these circumstances, the expected value of the parcel volume of a single receiver served by the $C F$ operator's vehicles will be $E(u)=\int_{0}^{u_{\max }} u f(u) d u$. On the contrary, each carrier will serve with the regular distribution network a total amount of $N_{i}\left(1-F\left(u_{\max }\right)\right)$ receivers whose parcel volume are $y>u_{\max }$ (like strategy $A$ ).

- $\quad$ It is assumed that each carrier $i(i=1, . ., M)$ has to pay a fare $\theta_{C F, i}\left(u_{k}\right)$ to the $C F$ operator for each parcel to be distributed through the $C F$. This fare may depend on the parcel volume $u_{k}(0 \leq$ $u_{k} \leq u_{\text {max }}$ ) in real agreements between CF operator and carriers, presenting an increasing function with regard to the parcel volume. For example, $\theta_{C F, i}\left(u_{k}\right)=\theta_{\min }+\hat{\theta} u_{k}$, where $\theta_{\text {min }}, \hat{\theta}$ are positive constants. However, for the sake of simplicity, we formulated the necessary conditions considering the equivalent flat fare $\theta_{C F}=\frac{\left\{\Sigma_{i=1}^{M} \Sigma_{k=1}^{N_{i}} \theta_{C F, i}\left(u_{k}\right)\right\}}{\left(N F\left(u_{\max }\right)\right)}$ regardless parcel volume.

Strategies $A$ and $B$ will be compared in terms of transportation costs, the externalities caused by the distribution system to the citizens and the net benefit of the $C F$ operator (only for strategy $B$ ). Throughout the paper, the superscripts $A, B, C F, R$ refer to the estimation of variables or parameters for independent carriers (strategy $A$ ), collaborating carriers through a consolidation facility (strategy $B$ ), the $C F$ operator and those parcels of regular carriers not routed through the consolidation facility, respectively. It is assumed that all the carriers try to minimize their costs. 

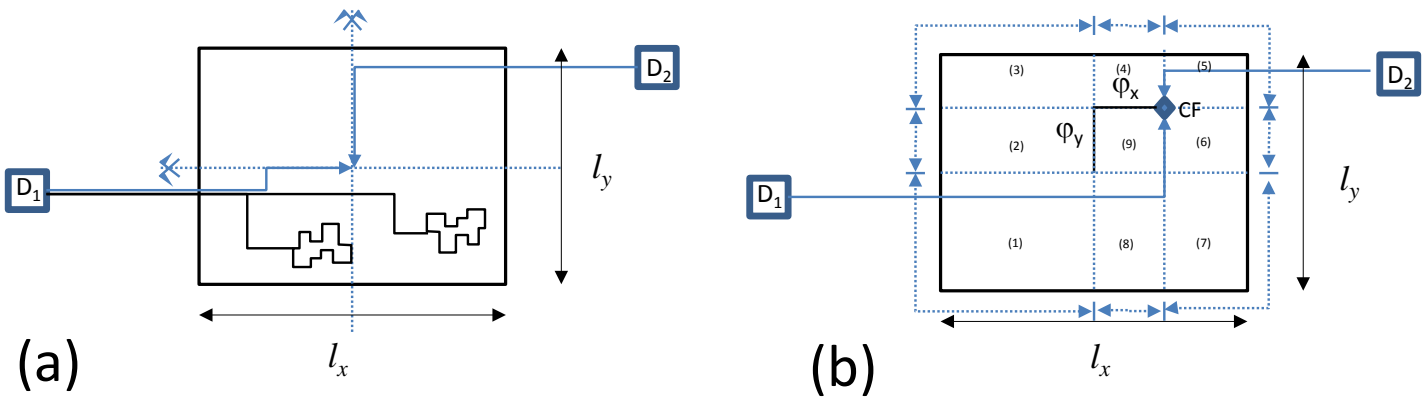

Figure 3. a) Scheme of regular distribution in Strategy $A$ and b) carriers' distribution through consolidation facility in Strategy $B$.

\subsubsection{Strategy A. Independent deliveries}

As mentioned before, in the regular distribution, each carrier is responsible for visiting its corresponding receivers independently with its own fleet. The total cost of the $M$ carriers $\left(Z_{C}^{A}\right)$ in strategy $A$ is the sum of the costs of each individual carrier $\left(Z_{C, i}\right)$ presented in Section 3.1 (see Equation 7). Moreover, the cost of the emissions produced by the whole freight fleet $\left(Z_{\text {ext }}^{A}\right)$ is addressed in Equation (8). Let $E$ be the different types of pollutants under analysis; $e_{j}(v)$ the emission factor of pollutant $j$ corresponding to the vehicle used by carriers (amount of pollutant $j$ /unit of distance) and the cruising speed of vehicle $v$; and $\$_{j}$ the monetary cost of the impact of an amount of pollutant $j$ on the society and the environment. Therefore, the total cost of the emissions caused by each kilometer run by a carrier vehicle at speed $v$ is estimated by $\varepsilon(v)=\sum_{j=1}^{E} e_{j}(v) \$_{j}$. This approach is consistent to the methodology presented in EMEPEEA (2013).

The estimation of the local and line-haul distance presented in Equations (9)-(10) depends on the relative market share of each specific carrier $i, \phi_{i}(i=1, . . M)$ and the maximal number of receivers that one vehicle can serve in one tour, $\psi_{i}^{A}=\min \left\{\frac{C}{E(y)} ; \frac{H-\beta / v_{L}}{\frac{2 \delta_{i}^{-1 / 2}}{\sqrt{3} v_{L}}+\tau}\right\}$. Here, the time horizon $H$ has been considered as the maximal time period in which regular carrier's vehicles are allowed to run within the city boundary. Therefore, we do not consider the running time in the line-haul distance (term $2 \rho / v_{L H}$ in the estimation of $\psi$ ) since this operation can happen outside the temporal restriction $H$. Note that in the estimation of local and line-haul distance, the number of tours are defined by $\left[\frac{N \phi_{i}}{\psi_{i}^{A}}\right]^{+}$, where $[x]^{+}$is a mathematical operator that gives the lowest integer value greater than or equal to $x$.

$$
\begin{gathered}
Z_{C}^{A}=\sum_{i=1}^{M} Z_{C, i}^{A}=\sum_{i=1}^{M}\left(c_{d}\left(D_{L, i}^{A}+D_{L H, i}^{A}\right)+c_{t} T_{i}^{A}\right) \\
Z_{e x t}^{A}=\sum_{i=1}^{M}\left(D_{L, i}^{A} \varepsilon\left(v_{L}\right)+D_{L H, i}^{A} \varepsilon\left(v_{L H}\right)\right) \\
D_{L}^{A}=\sum_{i=1}^{M} D_{L, i}^{A}=\frac{2}{\sqrt{3}}(A N)^{1 / 2} \sum_{i=1}^{M} \phi_{i}^{1 / 2}+\beta \sum_{i=1}^{M}\left[\frac{N \phi_{i}}{\psi_{i}^{A}}\right]^{+} \\
D_{L H}^{A}=\sum_{i=1}^{M} D_{L H, i}^{A}=2 \sum_{i=1}^{M} \rho_{i}\left[\frac{N \phi_{i}}{\psi_{i}^{A}}\right]^{+} \\
T_{A}=\left(D_{L}^{A} / v_{L}+D_{L H}^{A} / v_{L H}\right)+\tau N
\end{gathered}
$$

\subsubsection{Strategy B. Collaboration through a consolidation facility.}


The total transportation costs are divided into three distribution components: the costs that traditional carriers undergo to distribute goods to the consolidation facility $\left(Z_{C}^{B}\right.$, Equation 12$)$, the costs of the $C F$ operator for last-mile distribution $\left(Z_{C F}\right.$, Equation 13) and the cost incurred by carriers in a regular distribution to perform these deliveries $\left(Z_{C}^{R}\right.$, Equation 14$)$. The term $Z_{C}^{B}$ includes: the transportation cost associated to the link distribution center- $C F$ covered with regular carrier vehicles $\left(Z_{D T}^{B}\right)$, and the price that the carriers pay to the $C F$ operator for using the $C F\left(Z_{\theta}^{B}\right)$. The vehicles deployed in the line-haul link will carry all parcels of each company to be distributed in the area.

The cost analysis for the CF operator considers the cost concerning the distance covered, the travel time and a new term $\Omega$ that captures the investment cost to build and maintain the consolidation facility itself (see Equation 13). Parameter $\Omega$ is expressed in monetary units per unit of service time. Since the consolidation facility is not conceived to be a storage warehouse and parcels are shipped on the same day, the inventory cost of parcels is neglected. We assume that the cruising speed of the fleet used by $C F$ operator is equal to $v_{L}$. Although the distribution of $C F$ vehicles is performed within the area of service, we also consider a line-haul distance in this area due to the non-centralized location of the facility. Due to the potential lowest vehicle dimensions and city access restrictions, we consider that this fleet performs fewer detours in comparison to conventional vehicles used by regular carriers. Therefore, the corresponding detour distance for $C F$ fleet is assumed to be $\beta^{C F} \leq \beta$. In addition to that, those environmental-friendly vehicles may be exempted to obey the temporal access restriction imposed by local governments in local distribution. Therefore, the maximum distribution time horizon for $C F$ operator's vehicles is supposed to be $H^{C F}\left(H^{C F} \geq H\right)$. Here, we have also supposed specific unit cost $\left(c_{d}^{C F}, c_{t}^{C F}\right)$, emissions factors $e_{j}^{C F}(v)$ and the monetization of all emission per veh-km $\varepsilon^{C F}(v)=$ $\sum_{j=1}^{E} e_{j}^{C F}(v) \$_{j}$, as fleets can be adapted to the urban environment.

The transportation cost incurred by carriers to perform regular deliveries (without unloading them at the consolidation facility) is addressed in Equation (14), with the same assumptions considered in Strategy $A$. We assume that these tours can be performed with the same vehicles feeding the consolidation facility. Finally, Equation (15) takes into account the total cost of emissions to be considered in Strategy $B$.

$$
\begin{gathered}
Z_{C}^{B}=\sum_{i=1}^{M} Z_{D T, i}^{B}+\sum_{i=1}^{M} Z_{\theta, i}^{B}=\sum_{i=1}^{M}\left(c_{d} D_{L H, i}^{B}+c_{t} T_{i}^{B}\right)+\sum_{i=1}^{M} \theta_{C F} N_{i} F\left(u_{\max }\right) \\
Z_{C F}=\left(c_{d}^{C F}\left(D_{L}^{C F}+D_{L H}^{C F}\right)+c_{t}^{C F} T^{U}\right)+\Omega \\
Z_{C}^{R}=\sum_{i=1}^{M} Z_{C, i}^{R}=\sum_{i=1}^{M}\left(c_{d}\left(D_{L H, i}^{R}+D_{L, i}^{R}\right)+c_{t} T_{i}^{R}\right) \\
Z_{\text {ext }}^{B}=\sum_{i=1}^{M}\left(\left(D_{L H, i}^{B}+D_{L H, i}^{R}\right) \varepsilon\left(v_{L H}\right)+\left(D_{L}^{C F}+D_{L H}^{C F}\right) \varepsilon^{C F}\left(v_{L}\right)+D_{L, i}^{R} \varepsilon\left(v_{L}\right)\right)
\end{gathered}
$$

As we consider that the consolidation facility is located $\varphi=\varphi_{x}+\varphi_{y}$ away from the center of the distributing area, we assume that carriers will run an extra line-haul distance $\Delta \rho$. This variable $\Delta \rho$ may depend on the location of the carrier's distribution center outside the delivering area and consequently on the boundary of the distribution area through which the vehicles enter. Therefore, we divide the boundary of the region of service into eight segments of different length $l_{r}(r=1, . ., 8)$ to compute the average $\Delta \bar{\rho}$. In Figure $3 \mathrm{~b}$ the partition of the boundary of service area is plotted. The variable $\Delta \bar{\rho}$ may be estimated as the sum, for each segment of length $l_{r}$, of the product of the average incremental distance $\left(\Delta \rho_{\mathrm{r}}\right)$ and the probability $p_{r}$ of entering through the segment $r$ ( i.e. $\Delta \bar{\rho}=\Sigma_{r} \Delta \rho_{r} p_{r}$ ). As an example, the estimations of the corresponding values for segment $r=3$ are $\Delta \rho_{3}=\left(\varphi_{x}-\varphi_{y}\right)$ and $p_{3}=\frac{\frac{l_{y}}{2}-\varphi_{y}+\frac{l_{x}}{2}}{2\left(l_{x}+l_{y}\right)}$. With some algebra, the estimation of the average extra line-haul distance is $\Delta \bar{\rho}=\left[\varphi_{x}^{2}+\varphi_{y}^{2}\right] /\left(l_{x}+l_{y}\right)$.

The total distance in the line-haul distribution and the total travel time incurred by all carriers visiting the consolidation facility are estimated through Equations (16)-(17). In that case, the number of receivers 
that one carrier's vehicle tour is able to serve is estimated by $\psi^{B}=\left\{\frac{C}{E(y)}\right\}$. As vehicles do not perform local deliveries, we have only to satisfy $\left(H-2 \frac{\Delta \rho}{v_{L H}}-\tau^{\prime}\right) \geq 0$. In this constraint, we only subtract the amount of transport activities time spent inside the city. If this constraint is not guaranteed, there is not enough time to perform the line-haul distribution from the distribution center to the consolidation facility.

In Equation (17), we consider that each vehicle spends a total time $\tau$ due to the unloading operations of all parcels to be routed through $C F$. Although $\tau^{\prime}>\tau$, the total time of unloading operations is less when carriers drop all freight at the consolidation facility, i.e. $\tau^{\prime} N F\left(u_{\max }\right) E(y) / C \ll \tau N F\left(u_{\max }\right)$. On the other hand, the corresponding estimations of these variables for the vehicles of the $C F$ operator are presented in Equations (18)-(20). Here, the local distance formula is affected by a detour extra distance $\beta^{C F}$. For $C F$ operator's fleet, we also take into account an average line-haul distance $\overline{\rho_{u}}$ due to the decentered location of consolidation facility with regard to the center of the distribution area. In Figure $3 \mathrm{~b}$ we divide the service region into nine subregions of area $a_{q}(q=1, . ., 9)$.

The variable $\overline{\rho_{u}}$ is computed as the product of the average distance between the consolidation location and the central point of each subregion $q$, and, the probability that the local distance tour will be performed at subregion $q$ (i.e. $\overline{\rho_{u}}=\sum_{q} \rho_{u, q} p_{q}$ ). For subregion $q=3$, the expected value of line-haul distance is $\rho_{u, 3}=\left(\varphi_{x}+\frac{l_{x}}{4}+\left(\frac{l_{y}}{2}-\varphi_{y}\right) 1 / 2\right)$ and the corresponding probability is $p_{3}=\frac{1}{l_{x} l_{y}}\left(\frac{l_{x}}{2}\right.$. $\left.\left(\frac{l_{y}}{2}-\varphi_{y}\right)\right)$. Considering the 9 subregions involved, $\overline{\rho_{u}}=\left(l_{x}+l_{y}\right) / 4+\left(\varphi_{x}^{2} l_{x}+\varphi_{y}^{2} l_{y}\right) / l_{x} l_{y}$. In addition to this, the number of receivers visited in each tour is now compute by $\psi^{C F}=\min \left\{\frac{k_{C} C}{E(u)} ; \frac{\left(H^{C F}-\frac{\Delta \rho}{v_{L}}-\tau^{\prime}\right)-\frac{2 \overline{\rho_{u}}}{v_{L}}-\beta_{C F} / v_{L}}{\frac{2\left(\delta F\left(u_{\max }\right)^{-1 / 2}\right.}{\sqrt{3} v_{L}}+\tau^{C F}}\right\}$.

We consider that the time horizon available for the local distribution performed by $C F$ operator's fleet is affected by the time consumed by regular carriers in the link just between the distribution center - $C F$ and the unloading operation time. This assumption allows a proper comparison of strategy $A$ and $B$ in the same time period $H$ and it takes into account the transshipment operations at the consolidation facility. Finally, the line-haul distance, local distance and travel time of regular distribution of carriers to perform those shipments, whose volume is $y>u_{\max }$, is addressed in Equations (21)-(23) respectively. Note that the number of tours in regular distribution is estimated by $\psi_{i}^{R}=\min \left\{\frac{C}{E\left(y^{*}\right)} ; \frac{H-\frac{2 \Delta \rho}{v_{L H}}-\tau^{\prime}-\frac{2 \overline{\rho_{u}}}{v_{L}}-\frac{\beta}{v_{L}}}{\frac{2\left(\delta_{i}\left(1-F\left(u_{\max }\right)\right)^{-1 / 2}\right.}{\sqrt{3} v_{L}}}\right\}$, where $E\left(y^{*}\right)=\int_{u_{\max }}^{y_{\max }} y f(y) d y$. The line-haul distance covered by regular carriers for those parcels that will not be unloaded at $C F$ was already included in the term $D_{L H}^{B}$ of Equation (16). However, if the number of tours of each carrier $i$ arriving at $C F,\left[\frac{N \phi_{i}}{\psi_{i}^{B}}\right]^{+}$, is not sufficient to visit the corresponding $N \phi_{i}\left(1-F\left(u_{\max }\right)\right)$ receivers in a regular service, this carrier would have to dispatch more vehicles from the distribution center. The extra vehicles are estimated by the term $\Delta V_{i}$ in Equation (21). This situation can only happen when the time horizon $H$ for regular carrier is significantly constrained.

$$
\begin{gathered}
D_{L H}^{B}=\sum_{i=1}^{M} D_{L H, i}^{B}=\sum_{i=1}^{M} 2\left(\rho_{i}+\Delta \rho\right)\left[\frac{N \phi_{i}}{\psi_{i}^{B}}\right]^{+} \\
T^{B}=\sum_{i=1}^{M} T_{i}^{B}=\left(\frac{D_{L H}^{B}}{v_{L H}}\right)+\tau^{\prime} \sum_{i=1}^{M}\left[\frac{N F\left(u_{\max }\right) \phi_{i}}{\psi_{i}^{B}}\right]^{+} \\
D_{L}^{C F}=\frac{2}{\sqrt{3}}\left(A N F\left(u_{\max }\right)\right)^{1 / 2}+\beta^{C F}\left[\frac{N F\left(u_{\max }\right)}{\psi^{C F}}\right]^{+}
\end{gathered}
$$




$$
\begin{gathered}
D_{L H}^{C F}=2 \overline{\rho_{u}}\left[\frac{N F\left(u_{\max }\right)}{\psi^{C F}}\right]^{+} \\
T^{C F}=\frac{D_{L}^{C F}}{v_{L}}+\frac{D_{L H}^{C F}}{v_{L}}+\tau^{C F} N F\left(u_{\max }\right) \\
D_{L H}^{R}=\sum_{i=1}^{M} D_{L H, i}^{R}=2\left(\sum_{i=1}^{M} \Delta V_{i}\left(\rho_{i}+\Delta \rho\right)\right)
\end{gathered}
$$

Where

$$
\begin{gathered}
\Delta V_{i}=\max \left\{0 ;\left[\frac{N\left(1-F\left(u_{\max }\right)\right) \phi_{i}}{\psi_{i}^{R}}\right]^{+}-\left[\frac{N \phi_{i}}{\psi_{i}^{B}}\right]^{+}\right\} \\
D_{L}^{R}=\sum_{i=1}^{M} D_{L, i}^{R}=\frac{2}{\sqrt{3}}\left(A N\left(1-F\left(u_{\max }\right)\right)\right)^{1 / 2} \sum_{i=1}^{M} \phi_{i}^{1 / 2}+\left(\beta+2 \overline{\rho_{u}}\right) \sum_{i=1}^{M}\left[\frac{N\left(1-F\left(u_{\max }\right)\right) \phi_{i}}{\psi_{i}^{R}}\right]^{+} \\
T^{R}=\frac{D_{L}^{R}}{v_{L}}+\frac{D_{L H}^{R}}{v_{L H}}+\tau N\left(1-F\left(u_{\max }\right)\right)
\end{gathered}
$$

\section{Profitability Analysis}

In this section, the necessary conditions to ensure the profitability of the distribution through a Consolidation Facility $(C F)$ are addressed for each stakeholder involved. To do this, we compare the cost components provided for the distribution system with a consolidation strategy (referred as a strategy $B$ ) to the corresponding terms derived for the regular distribution without the provision of a $C F$ (referred as strategy A). In the following analysis, let $G_{c, i}^{A}, G_{c, i}^{B}$ and $G_{C F}$ be the gross revenues of carrier $i$ in operational strategy $A$, the gross revenues of carrier $i$ in strategy $B$ and the gross revenues of $C F$ operator, respectively. We used the same approach provided in Holguín-Veras (2008) when defining the necessary conditions for guarantying the efficiency of off-hour deliveries.

The first condition is to guarantee that the society receives benefits from the consolidated system, i.e. that the total logistic cost of the system and the externalities caused are reduced when carriers use the $C F$ service. Equation (24) defines the variable $\Delta_{1}$ as the sum of the benefits of all carriers in the strategy $B$ minus the corresponding benefits in strategy $A$, the benefits of $C F$ operator and the variation of the emissions' monetization. The latter is calculated as the amount of emissions saved in one day in Strategy B with regard to Strategy B ( $\mathrm{kg} /$ day), multiplied by the corresponding monetary value of one unit of pollutant. Variable $\Delta_{1}$ should be positive in order to ensure the efficiency of the collaboration of carriers from a system perspective. We assume that the carriers' revenues are maintained when they switch to the operation mode B (i.e. $G_{c, i}^{A}=G_{c, i}^{B}$ ), hence, receivers or suppliers will maintain the same fare $\theta_{c}$ in strategy $A$ and $B$. On the other hand, we assume that the revenues of the $C F$ operator come from the fare paid by those carriers using the consolidation facility and possibly other sources such as publicity or subsidies. The fares that carriers pay to the $C F$ operator are not relevant in this case. Since they appear in both, $C F$ operator gross revenues and carrier cost, they are cancelled. They are part of the cash flows among stakeholders. However, this constraint do not represent that each stakeholder is benefitted by the consolidation strategy implementation.

$$
\Delta_{1}=\left[\sum_{i=1}^{M}\left(G_{c, i}^{B}-Z_{c, i}^{B}-Z_{C, i}^{R}\right)-\sum_{i=1}^{M}\left(G_{c, i}^{A}-Z_{c, i}^{A}\right)\right]+\left(G_{C F}-Z_{C F}\right)+\left(Z_{\text {ext }}^{A}-Z_{\text {ext }}^{B}\right) \geq 0
$$

For this reason, the second condition to be fulfilled is to obtain a positive benefit of each carrier $i$ $(i=1, . ., M)$ involved in the new distribution system through the consolidation facility. In Equation (25), the variable $\Delta_{2 i}$ should be positive in order to ensure that those transportation cost savings of carrier $i$ are greater than the total fares to be paid to the $C F$ operator. The fare paid by carriers is included in the term $Z_{c, i}^{B}$. 


$$
\Delta_{2 i}=\left(G_{c, i}^{B}-Z_{c, i}^{B}-Z_{c, i}^{R}\right)-\left(G_{c, i}^{A}-Z_{c, i}^{A}\right) \quad \geq 0 \quad \forall i
$$

The conservative values of site-dependent parameters that satisfy the carrier profitability $\Delta_{2 i} \geq 0$ are constrained by Equation (26). The factors $A_{2, i}, B_{2, i}$ and $C_{2, i}$ are functions that depend on the demand of retailers $(N)$, the market share among carriers $\left(\phi_{i}\right)$ and vehicle capacities $\left(\psi_{i}\right)$. Therefore, once the latter terms have been defined, decision makers may prove that the rest of site-dependent parameters (vehicle unit cost, speeds, length between distribution centers and city center, stopping times and CF operation fare) satisfy Equation (26). The factors $A_{2, i}, B_{2, i}$ and $C_{2, i}$ are developed from Equations (7)-(23). They only capture distance and demand-related variables. Two vehicles with the same volumetric capacity may present different unit cost depending on the implementation instance. In fact, the distance- related unit cost parameter $c_{d}$ is different in fully- electric and combustion engine powered vehicles. In the latter, the consumer price of fuel products may differ in each country, depending on the fuel taxes or even government subsidies. The time-dependent cost also varies among sites according to the average driver salaries and vehicle renting/purchasing price.

It is interesting to identify the domains of $N_{i}$ and $F\left(u_{\max }\right)$ corresponding to values of factors $A_{2, i}, B_{2, i}$ and $C_{2, i}$ that will ever (or never) satisfy Equation (26), whatever the site-dependent parameters will be. This analysis is provided in Section 5.1.

$$
\left(c_{d}+\frac{c_{t}}{v_{L}}\right) A_{2, i}+\rho_{i}\left(c_{d}+\frac{c_{t}}{v_{L H}}\right) B_{2, i}+\tau c_{t} C_{2, i}-\theta \geq 0 \quad \forall i
$$

Where

$$
\begin{aligned}
& A_{2, i}=\frac{D_{L, i}^{A}-D_{L, i}^{R}}{N \phi_{i} F\left(u_{\max }\right)} \\
& =\left\{\frac{2}{\sqrt{3}}\left(A N \phi_{i}\right)^{\frac{1}{2}}\left(1-\left(1-F\left(u_{\max }\right)\right)^{\frac{1}{2}}\right)+\beta\left[\frac{N \phi_{i}}{\psi_{i}^{A}}\right]^{+}-\left(\beta+2 \overline{\rho_{u}}\right)\left[\frac{N\left(1-F\left(u_{\max }\right)\right) \phi_{i}}{\psi_{i}^{R}}\right]^{+}\right\} \\
& /\left\{N \phi_{i} F\left(u_{\max }\right)\right\} \\
& B_{2, i}=\frac{D_{L H, i}^{A}-D_{L H, i}^{B}-D_{L H, i}^{R}}{N \phi_{i} F\left(u_{\max }\right)}=\left\{2\left[\frac{N \phi_{i}}{\psi_{i}^{A}}\right]^{+}-2\left[\frac{N \phi_{i}}{\psi_{i}^{B}}\right]^{+}-2 \Delta V_{i}-2 \frac{\Delta \rho}{\rho_{i}}\left[\frac{N \phi_{i}}{\psi_{i}^{B}}\right]^{+}-2 \Delta V_{i} \frac{\Delta \rho}{\rho_{i}}\right\} /\left\{N \phi_{i} F\left(u_{\max }\right)\right\} \\
& C_{2, i}=\left\{N \phi_{i} F\left(u_{\max }\right)-\frac{\bar{\tau}}{\tau}\left[\frac{N \phi_{i} F\left(u_{\max }\right)}{\psi_{i}^{B}}\right]^{+}\right\} /\left\{N \phi_{i} F\left(u_{\max }\right)\right\}
\end{aligned}
$$

The third necessary condition is aimed at guaranteeing the net profitability of $C F$ operator, i.e. that the gross revenues of the $C F$ operator cover its costs $\left(G_{C F}-Z_{C F} \geq 0\right)$. In Equation (27) the variable $\Delta_{3}$ accounts for the benefits to run the consolidation service and the last mile distribution to the final receivers. It takes into account the corresponding costs of the operation as well as the investment cost associated to the implementation of a $C F$. Here, a given fare per parcel is charged to each carrier $i=1, . ., M$, that constitutes the major incomes of the $C F$ operator. However, we also consider the term $S_{C F}$ that captures all potential incomes per day coming from other sources than pricing. This term may represent subsidies paid by local governments or even incomes from advertising and publicity billboards located on both vehicles and the consolidation facility.

$$
\Delta_{3}=\left(\sum_{i=1}^{M} \theta_{C F} N_{i} F\left(u_{\max }\right)\right)+S_{C F}-c_{d}^{C F}\left(D_{L}^{C F}+D_{L H}^{C F}\right)-c_{t}^{C F} T^{C F}-\Omega \geq 0
$$

The corresponding $C F$ operator site -dependent parameters that result in a positive profitability for $C F$ operator $\left(\Delta_{3} \geq 0\right)$ must fulfill Equation (28) 


$$
+\left(S_{C F}-\Omega\right)-\left(c_{d}^{C F}+\frac{c_{t}^{C F}}{v_{L}}\right) A_{3}+\left(\theta_{C F}-c_{t}^{C F} \tau^{C F}\right) B_{3} \geq 0
$$

where

$A_{3}=D_{L}^{C F}+D_{L H}^{C F}=\frac{2}{\sqrt{3}}\left(A N F\left(u_{\max }\right)\right)^{1 / 2}+\beta^{C F}\left[\frac{N F\left(u_{\max }\right)}{\psi^{C F}}\right]^{+}+2 \overline{\rho_{u}}\left[\frac{N F\left(u_{\max }\right)}{\psi^{C F}}\right]^{+} ;$and $B_{3}=N F\left(u_{\max }\right)$

Finally, the last necessary condition is aimed at obtaining some positive environmental impact savings while the consolidation service is run. Although one may consider a wide list of potential externalities (noise, congestion, etc.); in this paper we only take into account the effect of GHG and local emissions on the environment and human health. Equation (29) defines the monetary value of the amount of emissions saved $\left(\Delta_{4}\right)$ due to the implementation of the consolidation strategy. The monetized emission parameters that determine a neutral effect $\left(\Delta_{4} \geq 0\right)$ in terms of emissions savings should satisfy Equation (30)

$$
\begin{gathered}
\Delta_{4}=\left(Z_{\text {ext }}^{A}-Z_{\text {ext }}^{B}\right) \geq 0 \\
+e \quad\left(v_{L}\right) \sum_{i=1}^{M} A_{4, i}+e \quad\left(v_{L H}\right) \sum_{i=1}^{M} \rho_{i} B_{4, i}-e^{C F}\left(v_{L}\right) C_{4} \geq 0
\end{gathered}
$$

where

$$
A_{4, i}=A_{2, i} N \phi_{i} F\left(u_{\max }\right) B_{4, i}=B_{2, i} N \phi_{i} F\left(u_{\max }\right) ; \text { and } C_{4}=A_{3}
$$

It can be easily demonstrated with some algebra that if the settings of this consolidation strategy satisfy a minimal profitability for all carriers, $C F$ operator and externalities, it also satisfies a positive total system profitability (Equation 24).

\section{Analysis of results}

In this section, the numerical estimations of the factors involved in Equations (26), (28) and (30) are analyzed for any generic distribution instance, in which we only determine the capacity of the vehicles used by carriers and CF operator. The distance and temporal-related unit cost of vehicles are not defined in advance, since they may vary according to the labor rules and energy cost in each country. The available gross vehicle typologies in the market $V c=\{$ van, pick-up, light truck, ... $\}$ constitute a discrete supply, where the volume capacity does not vary significantly for the type of vehicle chosen. These factor estimations are provided by retailer density- $F\left(u_{\max }\right)$ diagrams for a target vehicle capacity.

Moreover, a profitability analysis of a carrier-led consolidation strategy is addressed by means of formulas (24)-(25)-(27)-(29) in a specific problem instance. All input parameters related to the retailer demand and competition among carrier companies have been theoretically proposed. However, the sitedependent parameters (unit cost of vehicles, speeds, etc.) involved in Equations (26), (28), (30) have been defined resembling the current freight market situation in Barcelona city.

\subsection{Profitability analysis for a general instance}

In Appendix A2, the corresponding estimations for factors $A_{2, i}, B_{2, i}, C_{2, i}$ involved in Equation (26) for assessing carrier profitability are plotted in a $\left(\delta_{i}, F\left(u_{\max }\right)\right)$ diagram. The former factors are specific for the market share $\left(\delta_{i}=N \phi_{i}\right)$ of each carrier $i(i=1, . ., M)$ in the region of service and the vehicle capacity used. Here, we only developed the aforementioned estimations for volumetric capacities $C=9 \mathrm{~m}^{3}$ (representing small trucks, in Figures A1.a-A3.a) and $C=4.2 \mathrm{~m}^{3}$ (representing a commercial van, Figures A1.b-A3.b). The assumptions considered in the development of these estimations are the ones developed in Section 3.1-3.2 and the following: 
- In the expression of factor $C_{2, i}$, we conservatively assume a constant ratio $\frac{\bar{\tau}}{\tau}=5$. It represents the upper bound for the term $\frac{\bar{\tau}}{\tau}$ evaluated in a UCC pilot test conducted in Barcelona in 2013 (Navarro et al. 2016).

- A similar assumption has been defined for the term $\frac{\Delta \rho}{\rho_{i}}$. We suppose that the additional detour distance covered by carriers in order to visit the $C F$ operator is always less than the $10 \%$ of the total distance between city center and the location of carrier's headquarters. Here, we continue considering that distributions centers are always located out of the city. Therefore, the expression of $B_{2, i}$ was conservatively obtained for $\frac{\Delta \rho}{\rho_{i}}=0.10$.

- The other input parameters considered in the estimation of the aforementioned factors are $\beta=\beta^{C F}=0.10 \mathrm{~km}, \rho_{u}=2.83 \mathrm{~km}, y_{\max }=0.3 \mathrm{~m}^{3}$ and $E(y)=0.15 \mathrm{~m}^{3}$. In this subsection, we assume that the number of receivers that each vehicle is able to visit is not constrained by the temporal restrictions of the distribution system.

In order to satisfy Equation (26) for carrier $i$, one or many of the $A_{2, i}-B_{2, i}-C_{2, i}$ terms should be positive. However, there is a domain of $\left(\delta_{i}, F\left(u_{\max }\right)\right)$ pairs that produce negative values for all three factors. In fact, $B_{2, i}$ term is always negative while the $50 \%$ of the analyzed $\left(\delta_{i}, F\left(u_{\max }\right)\right)$ domain produces negative values for the $A_{2, i}$ term. The $C_{2, i}$ is the term that presents the smallest area of negative values in the domain of analysis. Interestingly, we identify a non-profit region of $\left(\delta_{i}, F\left(u_{\max }\right)\right)$ pairs for carrier $i$. It is equivalent to the $\left(\delta_{i}, F\left(\mathrm{u}_{\text {max }}\right)\right)$ domain providing negative values for factor $C_{2, i}$, plotted in Figure A.3a and A.3b in Appendix 2. This domain of $\left(\delta_{i}, F\left(u_{\max }\right)\right)$ variables always causes negative profit for carrier $i$ for any combination of cost coefficients $c_{d}, c_{t}, \tau$ and $\theta_{C F}$. This domain is identified for extremely low values of $F\left(u_{\max }\right)$ as well as low values of density of receivers served by this carrier $i\left(\delta_{i}\right)$. In fact, the negative domain of $\Delta_{2}$ is always obtained when $\delta_{i} \cdot F\left(\mathrm{u}_{\max }\right)<0.625$ for $C=9 \mathrm{~m}^{3}$ and $C=4.2 \mathrm{~m}^{3}$. However, this boundary only represents a lower bound of the non-profit domain. Obviously, we can also obtain unfeasible situations for $C_{2, i}>0$ (out of the former boundaries), especially when the fare $\theta_{C F}$ is a large number.

The values for factors $A_{3}-B_{3}$ of Equation (28) are plotted in a $\left(\delta, F\left(u_{\max }\right)\right)$ diagram. In Appendix A2, we show the corresponding diagrams for $C F$ operator vehicle capacity $C_{C F}=2.16 \mathrm{~m}^{3}$ (Figure A4.a and A5.a, representing a van) and $C_{C F}=1.2 \mathrm{~m}^{3}$ (Figures A4.b and A5.b, representing electric cargo bikes). In these cases, we plot the total density of receivers served by all carriers in the vertical axis. With some algebra and considering $\beta^{C F}=\overline{\rho_{u}}=0$, a lower bound of the carrier fare $\theta_{C F}$ that justifies the profitability for CF operator is defined in Equation (31).

$$
\theta \geq\left[c_{t}^{C F} \tau^{C F}+\left(c_{d}^{C F}+\frac{c_{t}^{C F}}{v_{L}}\right) \frac{2}{\sqrt{3 \delta_{i}}}\right]+\frac{\left(\Omega-S_{C F}\right)}{B_{3}} \quad \text { where } B_{3}=N F\left(u_{\max }\right)
$$

Finally, the analytical expressions of term $A_{4, i}-B_{4, i}-C_{4, i}$ in Equation (30) depend on $\left(\delta_{i}, F\left(u_{\max }\right)\right)$ pairs and they are quite similar to the former factors determined before $\left(A_{2, i}-B_{22}-A_{3}\right)$. Therefore, we do not exhibit the corresponding diagrams. From the analysis of $B_{4, i}$ and $C_{4, i}$ factors, we can state that $B_{4, i}<0$ and $C_{4, i}>0$. Therefore, if the monetized emissions factors of vehicles $\left(e\left(v_{L H}\right)\right.$ and $e\left(v_{L}\right)$ ) are greater than 0 , the only condition that may satisfy Equation (30) is that $\sum_{i=1}^{M} A_{4, i}>0$, i.e. $\sum_{i=1}^{M} A_{2, i}>0$. Therefore, the domain of $\left(\delta_{i}, F\left(u_{\max }\right)\right)$ providing negative values for $\sum_{i=1}^{M} A_{2, i}$ can not be considered feasible for environmental purposes. However, depending on the market share of each carrier taking part in the initiative, the corresponding values of $A_{2, i}$ may take positive or negative results. From the diagrams plotted for $A_{2, i}$ factor in Appendix 2 (Figures A1.a, .b), we can ensure that $A_{2, i}<0, \forall i$, when the percentage of parcels routed through $C F$ is less than a critical $F^{*}\left(u_{\max }\right)$ value. This critical value is $F^{*}\left(u_{\max }\right)=0.36$ when the carrier vehicle capacity is $C=9 \mathrm{~m}^{3}$, and $F^{*}\left(u_{\max }\right)=0.49$ for $C=4.2 \mathrm{~m}^{3}$. The former conditions represent a conservative lower bound of $F^{*}\left(u_{\max }\right)$, that is, the domain $F\left(u_{\max }\right) \leq F^{*}\left(u_{\max }\right)$ worsens the emissions economic impact of consolidation strategy with regard to Strategy A. Obviously, 
depending on $e\left(v_{L}\right), e\left(v_{L H}\right)$, and $e^{C F}\left(v_{L}\right)$ values, the consolidation strategy can not be still justified from environmental perspective when $F\left(\mathrm{u}_{\max }\right)>F^{*}\left(\mathrm{u}_{\max }\right)$.

\subsection{Profitability analysis for a test instance}

An ideal problem has been proposed in order to quantify the potential effects of consolidation initiatives on stakeholders involved and verify the application of general necessary conditions in a specific case. This problem is defined by a set of input parameters summarized in Appendix 1. It has three variants representing particular implementations. In all of them, we consider that there are 1,000 receivers scattered in a rectangular area $A=3 \cdot 2 \mathrm{~km}^{2}$, i.e. the receiver density is $\delta=166.6 \mathrm{rec} / \mathrm{km}^{2}$. The parcel volume ordered by each receiver is uniformly distributed in the domain $(0 ; 0.3] \mathrm{m}^{3}$. The upper bound is defined considering the maximal allowable packaging from a real carrier (express pallet dimensions from DHL, 2013). However, we consider that only those parcels whose volume is less than $u_{\max }=0.24 \mathrm{~m}^{3}$ (i.e. $\left.F\left(u_{\max }\right)=0.8\right)$ can be routed through the consolidation facility. Both $\delta$ and $F\left(u_{\max }\right)$ are crucial parameters that have been defined at significant values in their viable domain. In order to compare both strategies under the same conditions of operation, stop times and vehicle detours have been considered to be identical for regular carrier and $C F$ operator's fleet. We take into account that parcel services in the area under study are provided by $M=10$ different carriers companies with the following market share: $\phi_{1}=0.2$; $\phi_{2}=\phi_{3}=0.05$ and $\phi_{i}=0.1,(i=4, . ., 10)$. We consider that all carriers present a distribution center located at $\rho=20 \mathrm{~km}$ away from the urban distribution area. Regular carriers are supposed to deliver goods to receivers with vehicles whose capacity is $C=9 \mathrm{~m}^{3}$. These input parameters have been considered according to a UCC pilot test conducted in Barcelona in 2014 (SMILE, 2015). This $\rho$ parameter is the mean distance value between the gravity center of the delivering area and the distribution centers of 6 main third party logistics that took part in the pilot test. All distribution centers were located out of the urban layout of Barcelona. The corresponding unit distance and temporal cost $\left(c_{t}\right.$ and $\left.c_{d}\right)$ are chosen according to the information gathered from a regional survey to road carriers in Catalonia, Spain (Generalitat de Catalunya, 2015). Local and line-haul cruising speed parameters have been defined by usual values presented in historical centers of cities like Barcelona, $v_{L}=20 \mathrm{~km} / \mathrm{h}$ and $v_{L H}=60 \mathrm{~km} / \mathrm{h}$. The consolidation facility in all scenarios is supposed to be located 2 kilometers away from the center of distribution area $\left(\varphi_{x}=1 \mathrm{~km} ; \varphi_{y}=1 \mathrm{~km}\right)$. The installation of a consolidation facility and all structural cost are expressed by a parameter $\Omega=46 € / \mathrm{h}$. It is assumed an investment cost of 50.000 euros (infrastructure), a lifetime of 10 years and a maintenance cost of $12.000 € /$ year (SMILE, 2015). Moreover, a constant fare of $\theta_{C F}=1$ euros/parcel is assumed to be paid by carriers for each parcel delivered through the consolidation facility. For the sake of simplicity, we consider a common fare per parcel for all carriers involved. A constant service charge per parcel is commonly adopted in theoretical studies and real implementations (Janjevic and Ndiaye, 2016). Moreover, the $C F$ operator fleet has $H^{C F}=8$ hours to perform all the deliveries in the urban area where the time horizon for regular carriers $(H)$ is variable.

The specific parameters that generate the three scenarios of this problem are explained below. In Scenario 1 , the $C F$ operator's fleet consists of fully-electric vans of real capacity $k_{c} C=2.16 \mathrm{~m}^{3}\left(k_{c}=0.24\right)$. The unit distance cost has been estimated considering the energy consumption per $\mathrm{km}$ and the average price of the electricity $(\mathrm{kWh})$ in Spain. In this case, we assume that there is no temporal access restriction in the urban area for regular carriers ( $H^{C F}=H=8$ hours). In that case, the corresponding factors of Equation (26) for the carrier with highest market share are $A_{2,1}=0.0894, B_{2,1}=-0.005$ and $C_{2,1}=0.9062$, while the corresponding to the carrier of lowest market share $A_{2,2}=0.1763 B_{2,2}=-0.005$ and $C_{2,2}=0.8750$. The corresponding factors involved in Equation (28) are $A_{3}=351$ and $B_{3}=800$. Considering the former factors, the profitability of both carriers can be ensured in advance by Equation (26) for the given consolidation fare, speeds and unit vehicle costs. However, the $C F$ operator profitability is not justified, since the inequality of Equation (28) is not satisfied. In addition to that, the corresponding emission monetization factors are $e^{C F}\left(v_{L}\right)=0$ (fully electric vehicles), $e\left(v_{L}\right)=0.0069 \quad € / \mathrm{veh}-\mathrm{km}$ and $e\left(v_{L H}\right)=0.0055 € / \mathrm{veh}-\mathrm{km}$. The corresponding factors of Equation (30) are $\sum_{i=1}^{10} A_{4, i}=115$ and $\sum_{i=1}^{10} B_{4, i}=-80$, that guarantee the environmental profitability of the consolidation strategy. 
Scenario 2 considers that the $C F$ operator fleet consists of electric cargo-bikes with real capacity of $k_{c} C=$ $1.2 \mathrm{~m}^{3}\left(k_{c}=0.133\right)$. We still consider no temporal access restrictions for regular carriers, $\left(H^{C F}=H=8\right.$ hours). The new values for the factors involved in Equation (28) are $A_{3}=558.6$ and $B_{3}=800$, that result in a positive balance of Equation (28) for the new unit distance and temporal costs $c_{d}^{C F}=0.015 € / \mathrm{veh}-\mathrm{km}$ and $c_{t}^{C F}=15.54 € /$ veh-h. This consideration neither affects the environmental viability nor the regular carrier profitability.

In Scenario 3, we maintain the same $C F$ operator fleet as in Scenario 2. In this case, there is temporal access restriction for the regular carrier's vehicles. Several cities have promoted the implementation of these policies in the city center to heavy and light commercial vehicles. In rush commercial periods, freight vehicles are not allowed to enter the city center and/or perform load/unload operations. Therefore, carriers must perform their deliveries within a specific time window, experiencing higher transportation costs. This situation is addressed in this paper considering a narrower time horizon to perform deliveries. We assume that $H=2.5$ hours. However, this temporal restriction is not considered for $C F$ operator vehicles, since electric cargo-bikes are allowed to circulate even along sidewalks and pedestrian priority streets. This situation is not previously addressed by Equations (26), (28) and (30), since the factors of former Equations are only valid if the route length is not restricted by temporal constrains.

Finally, a sensitivity analysis is carried out in the three former subscenarios with regard to the key parameters that much strongly affect the results: the density of receivers, $\delta=N / A$, and the maximal parcel volume threshold that the $C F$ operator is allowed to serve $\left(u_{\max }\right)$. The terms in brackets in Appendix 1 represent the parameters domain explored in the sensitivity analysis. Finally, an economic analysis of the sustainable domain of the carrier's fare is addressed in section 5.2.2.

\subsubsection{Discussion}

The profitability indicators for this problem are analyzed in this section based on the results of Table 1. In the first two Scenarios, the total distance covered by the sum of carriers and $C F$ fleet in Strategy B (1258; $1412 \mathrm{~km})$ is higher than the distance covered in Strategy A (1079; $1079 \mathrm{~km})$. This increment is due to the higher distance covered within the distribution area. The reason is that the vehicle capacity of the $C F$ fleet is smaller ( $\psi^{A}=60$ receivers; $\psi^{C F}=18$ receivers in Scenario 1 and $\psi^{C F}=10$ receivers in Scenario 2). However, in Strategy $B$ the $61-71 \%$ of the distance travelled within the city is carried out by electric vehicles, causing less local and GHG emissions. In addition to that, the total number of hours of vehicles $\left(T^{B}+T^{C F}+T^{R}\right)$ in Strategy $B$ is reduced by $10 \%$ with regard to Strategy $A$ in Scenario 1 . The reason is the reduction of the number of stops of the carriers at receivers' locations $(\tau)$. Scenario 1 needs less resources and less transport distance to perform all deliveries in comparison to Scenario 2 due to a higher vehicle capacity.

Consolidation strategies do not improve the level of congestion of Scenario 1 and 2, as more kilometers are run by the total fleet. In Scenario 2, the distance travelled by CF vehicles $\left(V_{C F}\right)$ does not contribute to traffic congestion, since electric cargo-bikes are allowed to run along sidewalks and pedestrian-priority streets. However, it may generate several conflicts with pedestrians. Therefore, consolidation strategies basically improve the reduction of stop time at the receiver's location, causing less problems in load/unload parking facilities. Nevertheless, when we analyze the total transportation cost of the system instead of the distance and time consumed (cost incurred by carriers, $C F$ operator and externalities), Strategy $B$ is always more efficient than Strategy $A$. The transportation cost saving ranges between 8-13\% regarding Strategy $A$.

In Scenario 3, when temporal access restriction to the distribution area is constrained for carriers $(H=2.5 h)$, the distance covered by the whole fleet as well as the number of resources are now higher than Scenarios 1 and 2 in regular conditions (Strategy A). This is a reasonable result consistent with several research analyses (Holguín-Veras et al 2013, Quak and Koster, 2009 and Qureshi et al, 2009). Access restrictions usually increase the delivery cost of parcels in a city. In this problem, this increment is around $12.8 \%$ (comparison of total cost of Strategy A in scenario 3 with regard to scenario1). However, the 
consolidation strategy (strategy $B$ ) allows a total distance reduction with regard to regular distribution (1479 km in Strategy A and $1412 \mathrm{~km}$ in Strategy B), as well as the reduction of the total amount of time needed (84 h in Strategy $A$ and $78.2 \mathrm{~km}$ in Strategy $B$ ). Hence, consolidation strategy may contribute to alleviate the negative increment of mileage and, therefore, the emissions caused by regular carriers when access restriction is activated. In this scenario, the total cost in Strategy $B$ is reduced by $23 \%$ with regard to Strategy $A$. In all three scenarios, the necessary conditions to guarantee social, carrier and $C F$ operator profitability are ensured.

In all three subscenarios, we do not consider any subsidy or external income for the $C F$ operator, therefore the total $C F$ operator costs must be balanced by the fare incomes (i.e. $z_{C F}=z_{C F}^{\prime} \leq \theta$ ). In fact, if we just consider those deliveries served through the consolidation facility $\left(N \cdot F\left(\mathrm{u}_{\max }\right)=800\right.$ receivers $)$, the unit average cost saving for a collaborative carrier is $\overline{\Delta z^{\prime}}=\{1.218 ; 1.218 ; 1.556\} € /$ parcel in Scenarios 1, 2 and 3 respectively. In these situations, the corresponding $C F$ operator unit costs are $z_{C F}=z_{C F}^{\prime}=\{0.998$; $0.867 ; 0.867\} € /$ parcel. Therefore, even if carriers pay the proposed fare $\left(\theta_{C F}=1 € /\right.$ parcel), this situation ensures a net benefit for carriers of 0.218; 0.218 and $0.556 €$ /parcel routed through $C F$ in Scenarios 1, 2 and 3 respectively. The corresponding benefits for the $C F$ operator are 0.002; 0.133 and $0.133 € /$ parcel.

Table 1. Results in Scenarios

Scenario 1 Scenario 2 S cenario 3

Strategy A

\begin{tabular}{rrr} 
& & \\
\hline 278.9 & 278.9 & 279.9 \\
800.0 & 800.0 & 1200.0 \\
77.3 & 77.3 & 84.0 \\
2117.3 & 2117.3 & 2387.4 \\
6.3 & 6.3 & 8.5 \\
2123.6 & 2123.6 & 2395.8 \\
$\mathbf{2 . 1 1 7}$ & $\mathbf{2 . 1 1 7}$ & $\mathbf{2 . 3 8 7}$ \\
\hline & & \\
\hline 816.0 & 816.0 & 816.0 \\
18.4 & 18.4 & 18.4 \\
84.2 & 87.8 & 87.8 \\
187.5 & 337.5 & 337.5 \\
33.6 & 41.3 & 41.3 \\
170.7 & 170.7 & 170.7 \\
0.0 & 0.0 & 0.0 \\
18.5 & 18.5 & 18.5 \\
659.5 & 659.5 & 659.5 \\
800.0 & 800.0 & 800.0 \\
0.0 & 0.0 & 0.0 \\
798.2 & 693.6 & 693.6 \\
483.3 & 483.3 & 483.3 \\
5.6 & 5.6 & 5.6 \\
1946.6 & 1842.0 & 1842.0 \\
$\mathbf{1 . 1 4 3}$ & $\mathbf{1 . 1 4 3}$ & $\mathbf{1 . 1 4 3}$ \\
\hline & & \\
\hline 177.0 & 281.6 & 553.8 \\
10.5 & 10.5 & 37.5 \\
28.4 & 28.4 & 55.4 \\
1.8 & 106.4 & 106.4 \\
0.7 & 0.7 & 2.9 \\
0.975 & 0.975 & 1.245 \\
& & \\
$\mathbf{1 . 2 1 8}$ & $\mathbf{1 . 2 1 8}$ & $\mathbf{1 . 5 5 6}$ \\
1.263 & 1.263 & 1.938 \\
1.177 & 1.177 & 1.346 \\
0.998 & 0.867 & 0.867 \\
$\mathbf{0 . 9 9 8}$ & $\mathbf{0 . 8 6 7}$ & $\mathbf{0 . 8 6 7}$ \\
& & \\
& &
\end{tabular}

$€ /$ rec 


\subsubsection{Sensitivity analysis with regard to the number of receivers}

One of the major challenges to economically justify a carrier -led consolidation strategy (Strategy $B$ ) is the existence of a minimal demand for the consolidation facility. Let $\delta_{C F}$ be the spatial density of receivers that would be served through the consolidation facility, $\delta_{C F}=\delta F\left(u_{\max }\right)$, where $\delta=N / A$. Therefore, a sensitivity analysis of the profitability of each stakeholder involved with regard to receiver density and the maximal parcel volume admitted by $C F$ operator is presented in Figures 4-7. Figure 4 determines the social benefit $\left(\Delta_{1}\right)$ corresponding to the input parameters pairs $\left(F\left(u_{\max }\right)\right.$; $\left.\delta\right)$. The boundary among regions of different benefit corresponds to an isocurve of the benefit function of Equation (24), i.e. $\Delta_{1}=$ ct. The corresponding graphics of the benefits of carriers $\left(\Delta_{2}\right), C F$ operator $\left(\Delta_{3}\right)$ and environment $\left(\Delta_{4}\right)$ are depicted in Figure 5, 6 and 7 respectively. The discrete nature of the number of routes (integer variable) creates several discontinuities in these functions as it can be observed in the former Figures. In order to provide a more understandable analysis, we include in Figure 4 the exhibit (a), in which we calculate the corresponding economic condition in Scenario 1, considering a perfect continuous domain of the number of tours needed by the carriers and the $C F$ operator. We calculate the number of tours needed by stakeholder $x$ in Equations (9)-(10), (16)-(19), (21)-(22) by the real number provided by the quotient between the number of receivers visited $\left(N^{x}\right)$ and the maximal number of receivers that one vehicle would serve in one tour $\left(\psi^{x}\right)$. We refer to these estimations as the continuous version of the problem. Therefore, in Figure 4a, we replace $\left[N^{x} / \psi^{x}\right]^{+}$in the former equations by its corresponding real number $N^{x} / \psi^{x}$.

Figure 4a present an increasing benefit for society in the continuous version of the problem for larger $C F$ demand of deliveries $\delta_{C F}$, i.e. higher receiver densities $(\delta)$ and cumulative distribution $F\left(u_{\max }\right)$. The discrete versions (Figures $4 \mathrm{~b}, \mathrm{c}$ and d) present the same tendency in $\Delta_{1}$ although several discontinuities can be identified for given receiver densities. The society benefits in Scenario 2 (Figure 4c) are slightly higher than the corresponding value for Scenario 1 (Figure 4b, electric vans). However, from Figure 4d and 5b, it's realized that the society and carrier profitability in scenario 3 (temporal access restriction) significantly outperforms the previous values obtained in scenarios 1-2. The promotion of a consolidation facility can help local authorities reducing the extracost caused by the access restriction policy.

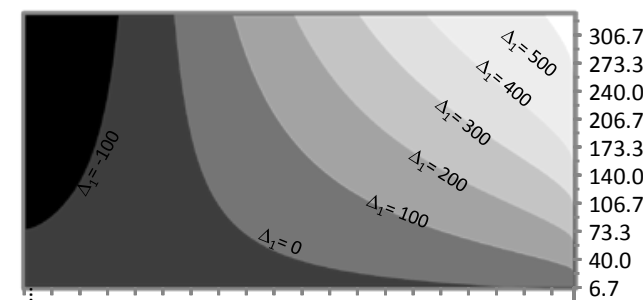

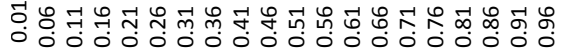

$F\left(u_{\max }\right)$

(a)

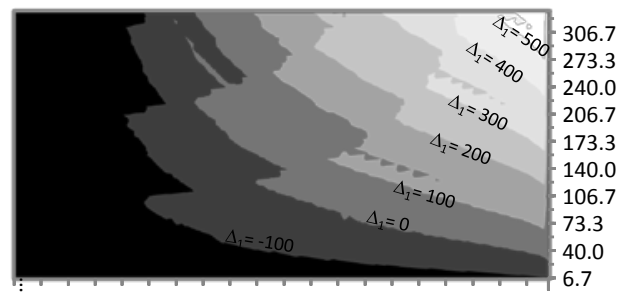

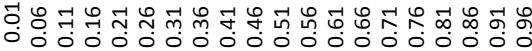
$F\left(u_{\max }\right)$

(b) 


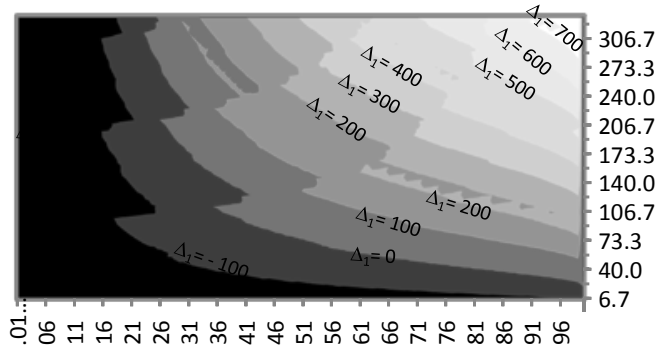

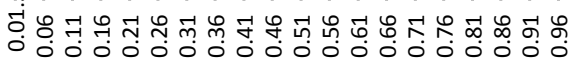

$F\left(u_{\max }\right)$

(c)

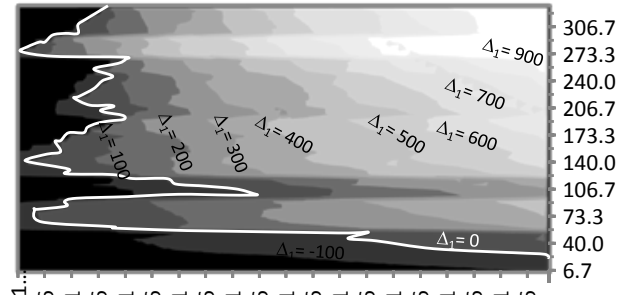

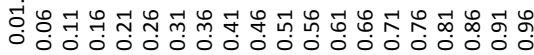

$F\left(u_{\max }\right)$

(d)

Figure 4. $\Delta_{1}$ Is ocurves (€/day) in Scenario 1 (a, b), Scenario 2 (c) and Scenario 3 (d). Exhibit (a) represents the continuous problem.

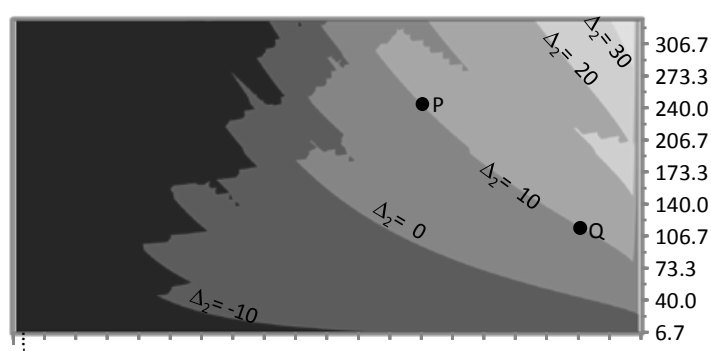

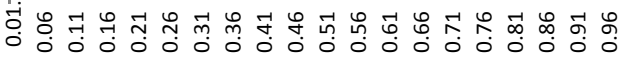

$\mathbf{F}\left(\mathbf{u}_{\max }\right)$

(a)

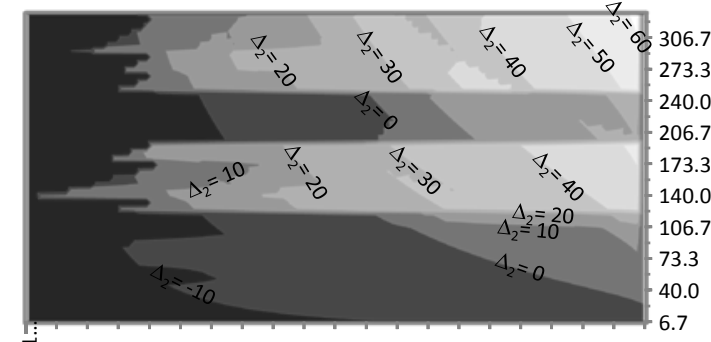

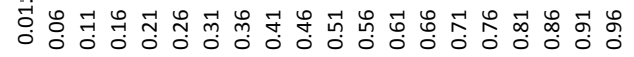

$F\left(u_{\max }\right)$

(b)

Figure 5. $\Delta_{2}$ isocurves ( $€$ /day) for the carrier with lowest profitability in Scenarios 1-2 (a) and Scenario 3 (b).

The society profitability presents negative domains of $\Delta_{1}$, that is, the operation in Strategy $A$ will cause lesser costs than Strategy $B$. In the analysis conducted, the required demand to ensure positive carrier profitability $\left(\Delta_{2}>0\right)$ is more restrictive than the corresponding for society. The domain size of $\left(F\left(u_{\max }\right) ; \delta\right)$ pairs where $\Delta_{2}>0$ depends on the carrier fare. If this fare was increased, a lower positive domain would be observed.

In Figure 5a, with the analysis of point $P$ and $Q$ as an example, we can see that point $P$, defined by $\left(F\left(u_{\max }\right) ; \delta\right)=(0.65 ; 247)$, presents a carrier profitability of $\Delta_{2}=10 €$. Point $Q,\left(F\left(u_{\max }\right) ; \delta\right)=(0.9 ; 120)$, also corresponds to the isocurve $\Delta_{2}=10 €$. However, in Point $P$ we need a higher number of parcels per area routed through $C F,\left(\delta_{C F}\right)_{P}=161 \mathrm{rec} / \mathrm{km}^{2}$, to ensure the same profitability of Point $\mathrm{Q},\left(\delta_{C F}\right)_{Q}=108$ $\mathrm{rec} / \mathrm{km}^{2}$. The reason is that a major fraction of parcels routed through consolidation facility (greater $\left.F\left(u_{\max }\right)\right)$ implies an increment of the average volume of these parcels. In this comparison, $E(u)_{Q}=0.135$ $\mathrm{m}^{3}$ and $E(u)_{P}=0.097 \mathrm{~m}^{3}$. These parcels of higher volume are served in Strategy $A$ by regular carriers, consuming a significant part of the potential vehicle capacity. In all scenarios, the maximal carrier cost savings correspond to the maximal density of receivers and the maximal value of $F\left(u_{\max }\right)$. A fact that deserves comment is the horizontal discontinuity curves appeared in Figure $5 \mathrm{~b}$. These lines correspond to values of a critical density of customers $(\delta *)$ in which the integer number of vehicles needed in Strategy A or B varies for the worst carrier. This fact causes that the carrier presenting the lowest cost saving is different for $\delta=\delta^{*}-\varepsilon$ and $\delta=\delta^{*}+\varepsilon(\varepsilon \rightarrow 0)$. This phenomenon is only identified in Scenario 3 when the vehicle capacity is highly constrained by $H$ (less $\psi_{i}^{A}, i=1, \ldots, M$ ). 


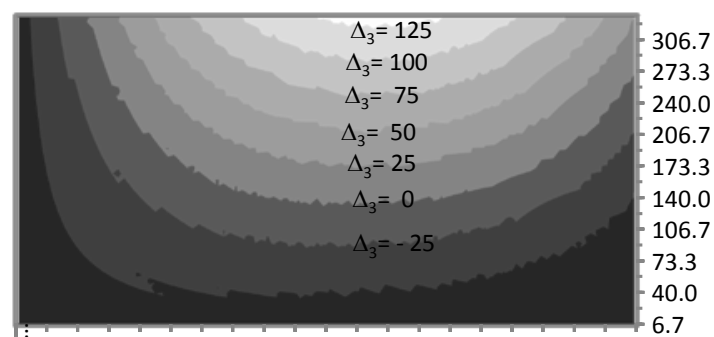

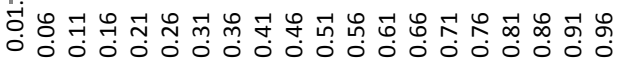

$$
\mathbf{F}\left(\mathbf{u}_{\max }\right)
$$

(a)

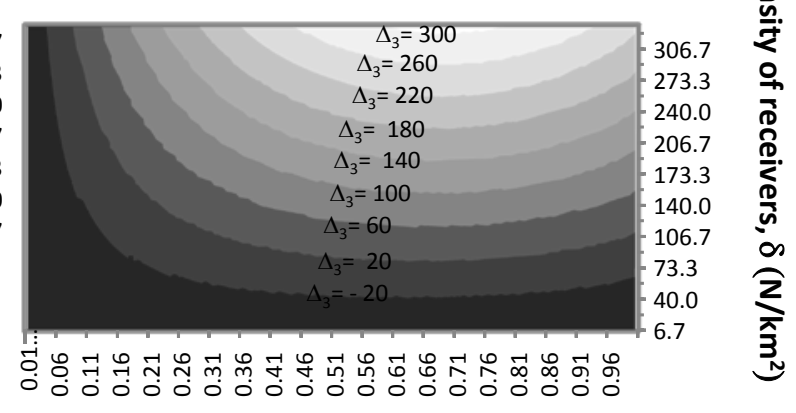

$F\left(u_{\max }\right)$

(b)

Figure 6. $\Delta_{3}$ isocurves (€/day) in Scenario 1 (a) and Scenarios 2-3 (b).

If we analyze Figure 6, the profitability of $C F$ operator does not follow the tendency explained for carriers. The maximal profitability is achieved for the maximum values of receiver densities studied $(\delta)$ and medium cumulative distribution $\left(\max \Delta_{3}=135.79\right.$ for $\delta=333.3 \mathrm{rec} / \mathrm{km}^{2}$ and $F\left(u_{\max }\right)=0.59$ in Scenario $1)$.

We identify two different behaviors of $\Delta_{3}$ boundaries depending on the value of $F\left(u_{\max }\right)$. For low values of $F\left(u_{\max }\right)$, the $\Delta_{3}$ isocurves correspond approximately to a constant value of the variable $\delta_{C F}$ in the $\left(F\left(u_{\max }\right), \delta\right)$ graphic-However, when $F\left(u_{\max }\right)>0.5$, it implies that parcels of higher volumes have to be served by $C F$ operator. As parameter $\psi^{C F}$ is constrained in these situations by the term $k_{c} C / E(u)$ where $0<u<u_{\max }$, larger $F\left(u_{\max }\right)$ implies lower values of $\psi^{C F}$. Consequently, more vehicles are needed to serve the demand, increasing the cost incurred by $C F$ operator. Finally, the behavior of $\Delta_{4}$ isocurves is analyzed in Figure 7. These isocurves are identical in Scenarios 1-and 2 since the CF operator vehicles are considered in both cases fully-electric. In those scenarios, if $F\left(u_{\max }\right)$ is less than 0.42 , the consolidation strategy always produces a higher monetization of the emission caused. In Scenario 3, the negative effects of the access restriction imposed are smoothed by the consolidation strategies. Generally, the positive emission savings domain is significantly higher than in the previous scenarios. In fact, the consolidation strategy would reduce transport emissions when $F\left(u_{\max }\right)>0.22$ and $\delta>130$ receivers $/ \mathrm{km}^{2}$. The monetized values of the emissions saved are the lowest component of the total social cost expressed in the variable $\Delta_{1}$. However, they constitute the most important justification of this measure for the livability of the city.

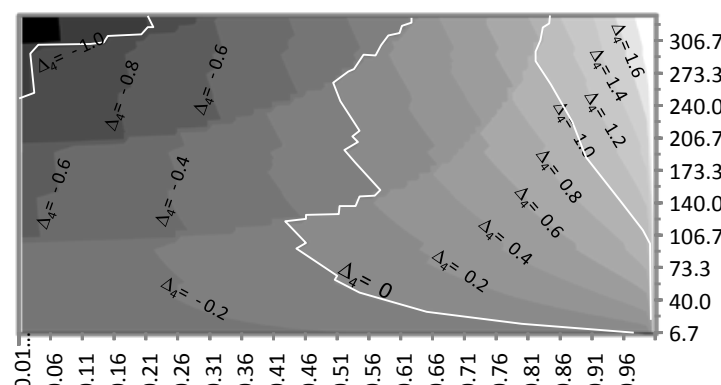

$F\left(u_{\max }\right)$

(a)

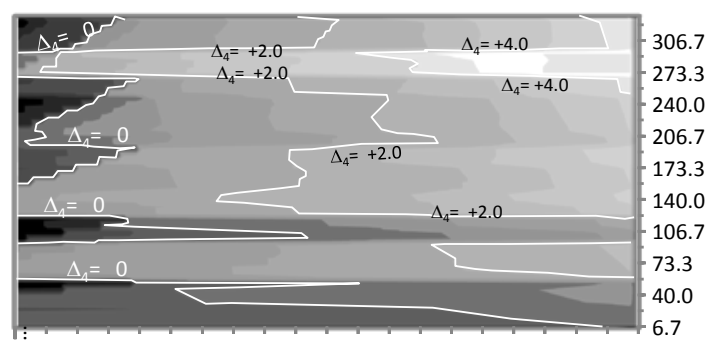

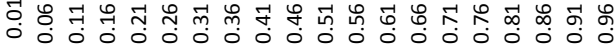
$F\left(u_{\max }\right)$

(b)

Figure 7. $\Delta_{4}$ isocurves (€/day) in Scenarios 1-2 (a) and Scenario 3 (b).

\subsubsection{Determination of the viable carrier's fare domain}


We are now trying to identify the domains of $\delta$ and $F\left(u_{\max }\right)$ in which we can set a fare $\theta_{C F}>0$ that guarantees a positive profitability of both carrier and $C F$ operator. Let $\eta_{r, i}=\frac{\left(z_{C, i *}^{A}-Z_{D T, i *}^{B}-Z_{C, i *}^{R}\right) /\left(N \phi_{i *} F\left(u_{\max }\right)\right.}{\left(Z_{C F}-S_{C F}\right) /\left(N F\left(u_{\max }\right)\right)}$. This variable is the ratio between the unit carrier cost savings per parcel distributed through the consolidation facility, and the unit net expenses of the $C F$ operator. We denote $i^{*}\left(i^{*}=1, \ldots M\right)$ as the carrier with the lowest cost savings when it changes from Strategy A to B. Therefore, this ratio $\eta_{r}$ represents whether there is a margin for a carrier to pay any fare that jointly ensures $\Delta_{2}>0$ and $\Delta_{3}>0$. When this ratio is $\eta_{r}>1$, the transportation cost savings incurred by the carrier $i^{*}$ are greater than the operating cost to serve the parcels of the $C F$ operator. Hence, the consolidation strategy is economically beneficial for both carrier $i^{*}$ and $C F$ operator. In fact, $C F$ operator could determine the service fare as $\theta_{C F}=\eta_{\theta} z_{C F}{ }^{\prime}$, where $\eta_{\theta}\left(1 \leq \eta_{\theta} \leq \eta_{r}\right)$ is the ratio between the $C F$ operator fare incomes and the corresponding unit expenses (excluding subsidies). The lower limit $\left(\eta_{\theta}=1\right)$ represents a perfect compensation between cost and fare revenues for the $C F$ operator. The upper limit $\left(\eta_{\theta}=\eta_{r}\right)$ determines that carrier with the lowest savings does not have any economic incentive to take part in the collaboration strategy since the total amount of transportation cost saving is equal to the cash flow paid to the $C F$ operator.

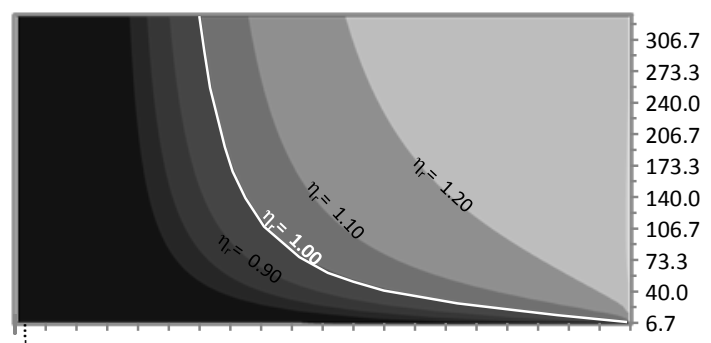

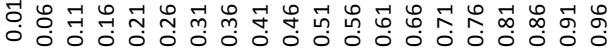

$F\left(u_{\max }\right)$

(a)

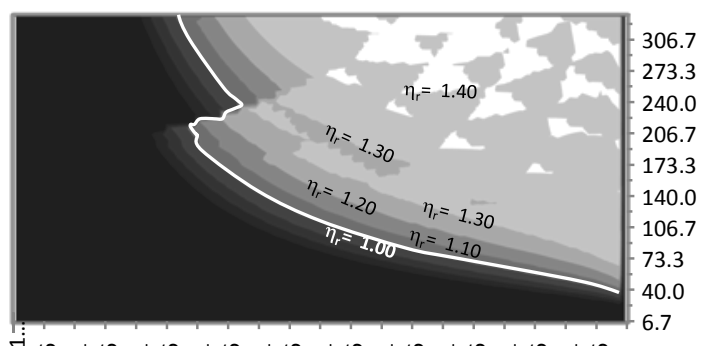

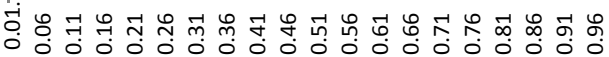

$F\left(u_{\max }\right)$

(c)

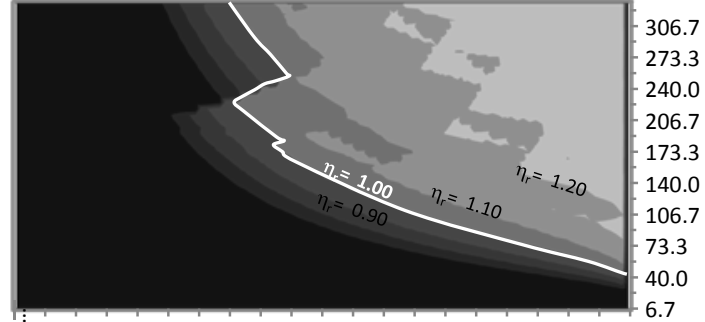

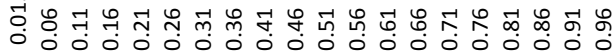

$F\left(u_{\max }\right)$

(b)

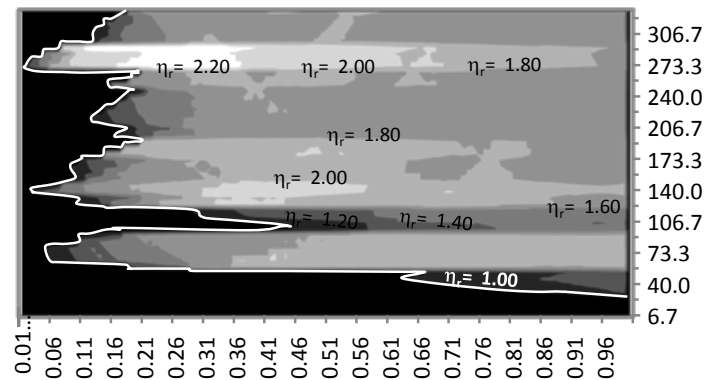

$F\left(u_{\max }\right)$

(d)

Figure 8. $\eta_{r}$-ratio for the carrier with the lowest profitability in Scenario 1 (a, b), Scenario 2 (c) and Scenario 3 (d). Exhibit (a) represents the continuous problem.

The $\eta_{r}$ ratio is evaluated as a function of $F\left(u_{\max }\right)$ and $\delta$ in Figure 8 for the previous scenarios considering the input data of Table 1. In Figure 8a (continuous problem version), the isocurves of $\eta_{r}$ ratio vary smoothly in the whole domain of analysis. On the contrary, in the discrete version of scenarios 1-2, the ratio presents discontinuities that determine a different system behavior. Two regions of similar behavior are identified, in which the isocurve $\eta_{r}=1.0$ is obtained by a roughly constant value of the density of receivers served through $C F\left(\delta_{C F}\right)$. In Scenario 1 , this critical density is $\delta_{C F} *=75 \mathrm{rec} / \mathrm{km}^{2}$ when $0<\delta<180$ $\mathrm{rec} / \mathrm{km}^{2}$ and $\delta_{C F} *=120 \mathrm{rec} / \mathrm{km}^{2}$ when $\delta<253 \mathrm{rec} / \mathrm{km}^{2}$. Similar behaviors can be identified for Scenario 2 . For both scenarios, less than the $50 \%$ of the total domain of $\left(F\left(u_{\max }\right), \delta\right)$ presents a value $\eta_{r}>1$. In Scenario 3, the $\eta_{r}$ ratio is ocurves present the oddest behavior due to the inclusion of the temporal access 
limitation constraint. Here, the domain where this ratio is $\eta_{r}>1$ encompasses the $85 \%$ of the total area of points $\left(F\left(u_{\max }\right) ; \delta\right)$ analyzed. For $\delta 140 \mathrm{rec} / \mathrm{km}^{2}$ and $F\left(u_{\max }\right)>0.2$, the parameter $\eta_{r}$ is always higher than 1 in Scenario 3.

\section{Conclusions}

Consolidation strategies can increase the efficiency of freight distribution in urban areas. However, the results that can be achieved and the organizational implications of consolidation strategies depend on the agent to whom these strategies are addressed. Carrier-led consolidation programs are addressed to those stakeholders that may experience logistic cost savings rather than to those that have the power to change the supply chain of goods. In fact, the participation of a low number of carriers (selection), each of them responsible for a high number of deliveries, may ensure the economic sustainability of the consolidation system.

In this paper, we analyze whether the potential cost savings incurred by regular carriers are sufficient to pay a fare to the Consolidation Facility $(C F)$ operator, i.e., if the unit carrier cost saving is higher than the unit consolidation cost. If this fact is achieved, a win-to-win collaboration can be proposed. For the sake of simplicity, we have assumed a constant fare per parcel for all carriers. The effects of this constant fare to carriers and $C F$ operator would be the same of linear fares with regard to parcel volumes, given a constant parcel volume distribution among receivers.

An analytical model was developed to identify the necessary conditions that guarantee positive benefits for society, carriers, CF operators and environment, when implementing a carrier-led consolidation. The model estimates the capital cost as well as the transportation cost of all agents involved by means of compact formulas for the consolidation and non-consolidation strategies. Given a vehicle capacity, there is a lower bound of the domain of receiver densities routed through the $C F$ (served by one carrier) that never produces a positive profitability for this carrier $\left(\delta_{\mathrm{i}} \cdot F\left(u_{\max }\right)<0.625 \mathrm{rec} / \mathrm{km}^{2}\right)$. In these situations, the consolidation strategy is not recommended. In the perspective of $C F$ operator, a lower bound of the fare per parcel to be paid by carriers has been identified. In real implementations, the carrier's fare must always be higher than this lower bound (given by Equation 31) that depends on the unit cost of $C F$ operator vehicle, local speed and time per stop. Finally, for environmental purposes, consolidation strategies increase the emissions associated to the transport system for $F^{*}\left(u_{\max }\right)<0.36$ when $C=9 \mathrm{~m}^{3}$ and for $F^{*}\left(u_{\max }\right)<0.49$ for $C=4.2 \mathrm{~m}^{3}$. The necessary condition to obtain positive emission savings is the most restrictive from the stated above. The former lower bounds prevail regardless the values of input sitedependent parameters such as time and distance-related unit costs, speeds, location of distribution centers, etc.

The formulas provided by the model are dependent on a given list of parameters describing the supply chains and two key decision variables: the carrier's fare and the maximal parcel volume accepted by the $C F$ operator. The carrier fare is just a cash flow to be paid among stakeholders and only affects the carrier and the $C F$ profitability. The maximal allowable parcel volume has to be fixed according to the parcel volume distribution and the capacity of the $C F$ operator's vehicles. The higher the maximal allowable parcel volume in the CF facility is set, the lesser average number of parcels delivered per tour. Therefore, if $C F$ operator increases the maximal admitted parcel volume, the corresponding incomes per tour are also reduced.

The profitability for carriers and $C F$ operator can be analyzed by means of the parameter $\eta_{r}$, i.e. the quotient between the unit carrier savings and the unit $C F$ operator cost (excluding subsidies or other incomes). When $\eta_{r}>1$, even if each carrier pays a fare equivalent to the unit cost per parcel of the $C F$ operator, its participation in the consolidation strategy is still economically positive.

In all numerical scenarios in which the analytical model has been tested for a given set of input parameters, there is a critical density of receivers served through $C F$ that ensures $\eta_{r}>1$. This critical density presents different behaviors depending on the relationship among the maximal parcel volume accepted by $C F$ operator and the maximal parcel volume in the area. In spite of this, for a general case of 
implementation in which we choose the most restrictive value, the critical density that satisfies the condition $\eta_{r}>1$ is $\delta_{C F}{ }^{*}=120 \mathrm{rec} / \mathrm{km}^{2}$ for the scenario where the capacity of electric vans is $2.4 \mathrm{~m}^{3}$. When $C F$ operator chooses electric cargo-bikes of $1.2 \mathrm{~m}^{3}$ capacity, the condition $\eta_{r}>1$ is achieved for critical densities of $\delta_{C F} *=93 \mathrm{rec} / \mathrm{km}^{2}$. The situations under which $C F$ operator and carrier are guaranteed $\left(\eta_{r}>1\right)$ are more restrictive than the global profitability constraint (total cost savings).

One of the facts that mostly affect the profitability of each stakeholder is the existence of a temporal access restriction. The results have demonstrated that the implementation of access restrictions increases the transportation cost of carriers and emissions in regular conditions (without CF). However, the implementation of the consolidation strategy measure may alleviate the negative impact of access restriction on carriers. Therefore, consolidation strategies may be a solution for those parcels that, because of the mandatory distribution time given by receivers, would be distributed when the access restriction is activated for regular carriers. The cost variations caused by the temporal restriction horizon $(H)$ are much more important than the ones caused by the vehicle type (vans or electric cargo-bikes). In the numerical instances, we consider that the $C F$ operator operated the local network with electric cargo-bikes, which has less unit vehicle cost. This fleet would have lesser impact on traffic since it can run along sidewalks and traffic calming streets. However, it may increase safety problems due to the coexistence with pedestrian flows. An interesting conclusion of our study is that the promotion of CF (UCC or uSA) does not necessary reduce the travel time and distance run by the gross commercial fleet. Therefore, as an opposite point to most guidelines, a general implementation of a consolidation facility does not always have to be justified as a policy driver to reduce traffic congestion. Therefore, the analytical model presented here will provide a useful tool to assess these variables and determine the potential impact on the traffic performance.

\section{Acknowledgments}

Authors would like to give special thanks to the management staff of the Municipality of Barcelona, Vanapedal (CF operator) and Mr. Simon Hayes for all their comments and advice. We would also like to thank the four anonymous reviewers for their valuable comments.

\section{References}

Allen, J., Browne, M., Woodburn, A., Leonardi, J., 2012. The role of urban consolipñdation centres in sustainable freight transport. Transp. Rev. 32 (4), 473-490.

Browne, M., Sweet, M., Woodburn, A., Allen, J., 2005. Urban freight consolidation centres. Transport Studies Group. University of Westminster. Final report.

Browne, M., Woodburn, A., Allen, J., 2007. The role of urban consolidation centres for different business sectors. In the 11th World Conference on Transport Research.

Campbell, J. F., 1990. Freight consolidation and routing with transportation economies of scale. Transportation Research Part B: Methodological, Volume 24, Issue 5, Pages 345-361.

Chen, Q., J. Lin, and K. Kawamura, 2012. Comparison of Urban Cooperative Delivery and Direct Delivery Strategies. Transportation Research Record: Journal of the Transportation Research Board, No.2288, Transportation Research Board of the National Academies, Washington, D.C., pp. 28-39.

Daganzo C. F. 1984a. The distance traveled to visit N points with a maximum of C stops per vehicle: An analytical model and an application. Transp. Sci. 18:4, 331-350.

Daganzo C. F. 1984b. The length of tours in zones of different shapes. Transpn. Res. 18B, 135-145.

Daganzo, C.F., 1988a. A comparison of in-vehicle and out-of- vehicle freight consolidation strategies. Transportation Research Part B: Methodological, Volume 22, Issue 3, 1988, Pages 173-180 
Daganzo, C.F., 1988b. Shipment composition enhancement at a consolidation center. Transportation Research Part B: Methodological, Volume 22, Issue 2, 1988, Pages 103-124.

Daganzo, C.F., 2005. Logistics system analysis. Springer.

DHL (2013). DHL express packaging guide. Protect your shipment with good and quality packaging. http://www.dhl.com.sg/content/dam/downloads/sg/express/shipping/dhl express packaging g uide 072013.pdf. Accessed on January 2016.

European Comission, 2005. Damages per tonne emission of PM2.5, NH3, SO2, NOx and VOCs from each EU25 Member State (excluding Cyprus) and surrounding seas. DG Environment. Available online: http://ec.europa.eu/environment/archives/cafe/activities/pdf/cafe cba externalities.pdf

EMEP/EEA, 2013. EMEP/EEA air pollutant emission inventory guidebook - 2013. Available online: http://www.eea.europa.eu//publications/emep-eea-guidebook-2013, Accessed January 2016.

Generalitat de Catalunya, 2015. Observatori de costos del transport de mercaderies per carretera a Catalunya, Butlletí de transports, Núm. 72 - setembre 2015. In catalan.

Gonzalez-Feliu, J. and Morana, J., 2011. Collaborative transportation sharing: from theory to practice via a case study from France. In Yearwood, J.L. and Stranieri, A. (eds.), Technologies for Supporting Reasoning Communities and Collaborative Decision Making: Cooperative Approaches, Information Science Reference, Hershey, pp. 252-271.

Holguín-Veras, J., Silas, M., Polimeni, J., 2008. An investigation into the attitudinal factors determining participation in cooperative multi-carrier delivery initiatives. In: Taniguchi, E., Thomson, R. (Eds.), Innovations in City Logistics IV. Nova Science Publishers, pp. 55-68.

Holguín-Veras, J. , 2008. Necessary Conditions for Off-Hour Deliveries and the Effectiveness of Urban Freight Road Pricing and Alternative Financial Policies in Competitive Markets. Transportation Research Part A: Policyand Practice, Vol. 42, No. 2, pp. 392-413.

Holguín-Veras, J., C.A. Torres, and X. Ban, 2013. On the Comparative Performance of Urban Delivery Vehicle Classes. Transportmetrica A: Transport Science, Vol. 9, No. 1, pp. 50-73.

Holguín-Veras, J. , Sánchez-Díaz, I., 2016. Freight Demand Management and the Potential of ReceiverLed Consolidation programs. Transportation Research Part A 84, 109-130.

Holroyd E. M. (1965). The optimum bus service: a theoretical model for a large uniform urban area. In L. C. Edie, R. Herman, and R. Rothery (Eds.), Vehicular Traffic Science, Proceedings of the 3rd International Symposium on the Theory of Traffic Flow. New York, Els evier.

Janjevic, M., Kaminsky, P., Ballé Ndiaye, A., 2013. Downscaling the consolidation of goods - state of the art and transferability of micro-consolidation initiatives. European Transport 54, Paper $n^{\circ} 4$.

Janjevic, M., Ndiaye, A. 2016. Investigating the theoretical cost-relationships of urban consolidation centres for their users. Transport. Res. Part A, in press, http://dx.doi.org/10.1016/i.tra.2016.10.027.

Kawamura, K. and Y. Lu., 2008. Evaluation of delivery consolidation in U.S. urban areas with logistics cost analysis. In Transportation Research Record: Journal of the Transportation Research Board, Transportation Research Board of the National Academies, Washington, D.C., 2007, pp. 34-42.

Kin, B., Verlinde, S., van Lier, T., and Macharis, C., 2016. Is there Life After Subsidy for an Urban Consolidation Centre? An Investigation of the Total Costs and Benefits of a Privately-initiated Concept. Transportation Research Procedia, Tenth International Conference on City Logistics, Spain 12, 357-369.

Köhler, U., 2001. City logistics in Germany. City Logistics II, pp. 203-214, Japan. Institute of Systems Science Research. 
Krajewska, M. and H. Kopfer, 2006. Collaborating freight forwarding enterprises. OR Spectrum, Vol. 28, No. 3, pp. 301-317.

Krajewska, M. A., H. Kopfer, G. Laporte, S. Ropke, and G. Zaccour, 2008. Horizontal cooperation among freight carriers: request allocation and profit sharing. The Journal of the Operational Research Society, Vol. 59, No. 11, pp. 1483-1491.

Lebeau, P., Verlinde, S., and Macharis, C. 2015. How authorities can support Urban Consolidation Centres? A review of the Best Practices. In Nectar Cluster 3: City Logistics and Sustainable Transport workshop.

Nathanail E., Gogas M., Papoutsis K., 2012. Urban freight and urban-interurban interfaces Best practices, implications and future needs. Deliverable D2.1 STRAIGHTSOL - STRAtegies and measures for smarter urban freIGHT SOLutions.

Newell G.F., C.F. Daganzo, 1986. Design of multiple-vehicle delivery tours. I. A ring-radial network. Transportation Research Part B: Methodological, 20(5), p.345-363.

Paddeu D., Fadda P., Fancello G., Parkhurst G., Ricci M., 2014. Reduced Urban Traffic and Emissions within Urban Consolidation Centre Schemes: The Case of Bristol. Transportation Research Procedia, Volume 3, p. 508-517

Quak, H.J. and M.B.M. de Koster, 2009. Delivering Goods in Urban Areas: How to Deal with Urban Policy Restrictions and the Environment. Transportation Science, Vol. 43, No. 2, pp. 211-227.

Qureshi, A.G., E. Taniguchi, and T. Yamada, 2009. An Exact Solution Approach for Vehicle Routing and Scheduling Problems with Soft Time Windows. Transportation Research Part E, Vol. 45, pp. 960-977.

Robuste, F., Daganzo C.F. and R.R. Souleyrette, 1990. Implementing vehicle routing models. Transportation Research Part B 24(4), pp.263- 286.

Roca-Riu, M. and M. Estrada, 2012. An Evaluation of Urban Consolidation Centers through Logistics Systems Analysis in Circumstances where companies have equal market shares. Procedia - Social and Behavioral Sciences, Vol 39, pp. 796-806. Special issue of the Seventh International Conference on City Logistics, Mallorca, Spain.

Roca-Riu, M., M. Estrada and E. Fernández, 2016. An evaluation of urban consolidation centers through continuous analysis with non-equal market share companies. Transportation Research Procedia, Vol 12, p. 370-382. Special issue of the Ninth International Conference on City Logistics, Tenerife, Spain.

Saberi, M. and Verbas, O. , 2012. Continuous Approximation Model For the Vehicle Routing Problem for Emissions Minimization at the Strategic Level. J. Transp. Eng., Vol. 138, No. 11, pp. 1368-1376.

SENDECO2, 2016. European Bourse for European Unit Allowancs and Carbon Credits. www.sendeco2.com. Accessed January, 2016.

SMILE, 2015. SMart green Innovative urban Logistics Models for Energy efficient mediterranean cities project), funded by the MED Programme. http://smile-urbanlogistics.eu/ Accessed on 1st January 2015.

SUGAR, 2011. Sustainable Urban Goods Logistics Achieved by Regional and Local Policies. City Logistics Best Practices: a Handbook for Authorities. www.sugarlogistics.eu. European Union.

UNFPA (2007). State of the World 2007- Unleashing the Potential of Urban Growth. United Nations Population Funds.

UNFPA (2012). Population Matters for Sustainable Development. United Nations Population Funds. 
Zhou, G., Van Hui Y., Liang L., 2011. Strategic alliance in freight consolidation. Transportation Research Part E 47 18-29. 


\section{Appendix 1: Glossary}

A glossary of terms used in the methodological approach and test instances is presented:

\begin{tabular}{|c|c|c|}
\hline$\overline{\text { Notation }}$ & Concept & Numerical values considered \\
\hline \multicolumn{3}{|c|}{ Demand and city input } \\
\hline$N$ & Number of receivers (deliveries in a day) & $1000(100-2000)$ \\
\hline$l_{x}, l_{y}$ & Major and minor rectangle side length, $(\mathrm{km})$ & 3,2 \\
\hline$A$ & Area of the city $\left(\mathrm{km}^{2}\right)$ & 6 \\
\hline$\delta$ & Density of receivers per unit of area & 166.67(16.67-333.33) \\
\hline$y_{\max }$ & Maximal parcel volume allowed by carriers in regular distribution $\left(\mathrm{m}^{3}\right)$ & 0.3 \\
\hline$u_{\max }$ & Maximal parcel volume allowed by CF operator, $\left(\mathrm{m}^{3}\right)$ & $0.24(0-0.3)$ \\
\hline$y_{k}$ & Volume of the parcel requested by each receiver $k(k=1, \ldots, N)$ & - \\
\hline$F(y)$ & Cumulative distribution function of parcels where volume $y_{k} \leq y_{\max }$ & - \\
\hline$f(y)$ & Probability density function of parcel volume & - \\
\hline$u_{k}$ & $\begin{array}{l}\text { Volume of the parcel requested by each receiver } k(k=1, \ldots, N) \text { served } \\
\text { through CF facility }\end{array}$ & - \\
\hline$\chi_{L}, \chi_{C F}$ & $\begin{array}{l}\text { Regulation measures imposed by city council to carrier vehicles and CF } \\
\text { operator vehicles respectively }\end{array}$ & \\
\hline$H$ & Available time period for regular carriers to deliver parcels to receivers, (h) & 8 (Scenario 1,2), 2.5 (Scenario 3) \\
\hline$H^{C F}$ & Available time period for CF op erator to deliver parcels to receivers, (h) & 8 \\
\hline \multicolumn{3}{|c|}{ Carrier input } \\
\hline$M$ & Number of carrier companies serving the area & 10 \\
\hline$\phi_{\mathrm{i}}$ & Market share of each carrier $i(i=1, . ., M)$ & $\begin{array}{c}\phi_{1}=0.2, \phi_{2}=\phi_{3}=0.05, \phi_{\mathrm{i}}=0.1 \\
(i=4, ., 10)\end{array}$ \\
\hline $\begin{array}{l}N_{i} \\
\delta\end{array}$ & $\begin{array}{l}\text { Number of retailers to be visited by carrier } i(i=1, . . M),\left(N_{i}=\phi_{i} N\right) \\
\text { Density of retailers served by carrier } i(i=1, . . M),\left(\mathrm{rec} / \mathrm{km}^{2}\right)\end{array}$ & - \\
\hline $\begin{array}{c}\sigma_{i} \\
\rho_{i}\end{array}$ & $\begin{array}{l}\text { Distance between the distribution center and the central point of the } \\
\text { delivering area, }(\mathrm{km})\end{array}$ & 20 \\
\hline$V_{C}, \theta_{C}$ & $\begin{array}{l}\text { Typology of vehicles used by carriers and transportation fare paid by } \\
\text { suppliers/receivers to carriers respectively }\end{array}$ & \\
\hline$c_{t}$ & Unit temporal cost of regular carrier vehicle, $(€ / \text { veh-h })^{* 1}$ & 23.49 \\
\hline$c_{d}$ & Unit distance cost of regular carrier vehicle, $\left(€ /\right.$ veh-km) ${ }^{* 1}$ & 0.280 \\
\hline C & Volume capacity of regular carrier vehicle, $\left(\mathrm{m}^{3}\right)^{* 1}$ & 9 \\
\hline$v_{L}, v_{L H}$ & $\begin{array}{l}\text { Local speed of regular carriers within the service area, line-haul speed of } \\
\text { regular carriers from distribution center to the service area, }(\mathrm{km} / \mathrm{h})\end{array}$ & 20,60 \\
\hline$\tau$ & Stop time per receiver in regular carriers distribution, (h) & 0.05 \\
\hline$\beta$ & Detour distance run by regular carriers per tour, (km) & 0.10 \\
\hline$\tau^{\prime}$ & $\begin{array}{l}\text { Stop time of carrier vehicle at CF due to the unloading operations of all } \\
\text { parcels to be routed through } C F,(\mathrm{~h})\end{array}$ & 0.25 \\
\hline \multirow{2}{*}{$\begin{array}{l}e_{j}\left(v_{L}\right) \\
e_{j}\left(v_{L H}\right)\end{array}$} & $\begin{array}{l}\text { emission factor of pollutant } j \text { corresponding to the carrier vehicle at } \\
\text { cruising speed } v \text { (local) and } v_{U H} \text { (linehaul), (g of pollutant } j / v e h-k m \text { ) }\end{array}$ & \\
\hline & $\begin{array}{l}\left.\mathrm{CO}_{2} / \text { NOx / PM 2.5 Emission factor (g/veh-km) }\right)^{* 2} \text { in local distribution } \\
\mathrm{CO}_{2} / \text { NOx / PM 2.5 Emission factor (g/veh-km) }{ }^{* 2} \text { in line-haul distribution }\end{array}$ & $\begin{array}{c}293.32 / 0.724 / 0.00188 \\
193.026 / 0.626 / 0.00089\end{array}$ \\
\hline \multicolumn{3}{|c|}{ CF operator input } \\
\hline$\varphi_{i x,} \varphi_{i y}$ & Distance from $C F$ location and central area in x, y coord., (km) & 1,1 \\
\hline$\delta_{C F}$ & Density of receivers whose parcel is routed by $C F$, $\left(\mathrm{rec} / \mathrm{km}^{2}\right)$ & \\
\hline$c_{t}^{C F}$ & Unit temp oral cost, (€/veh-h) ${ }^{* 1}$ & 22.14 (Scen. 1), 15.54 (Scen.2,3) \\
\hline$c_{d}^{C F}$ & Unit distance cost, (€/veh-km) & 0.032 (Scen. 1), 0.015 (Scen.2,3) \\
\hline$k_{C}$ & Capacity factor reduction & 0.24 (Scen.1), 0.133(Scen.2-3) \\
\hline$C_{C F}$ & Volume capacity of CF op erator vehicles, $\left(\mathrm{m}^{3}\right) C_{C F}=k_{C} C$ & 2.16 (Scen.1), 1.20 (Scen.2-3) \\
\hline$\beta^{C F}$ & $\begin{array}{l}\text { Detour distance run by CF operator vehicle in each tour due to the } \\
\text { allowable direction of street, }(\mathrm{km})\end{array}$ & 0.10 \\
\hline$\Omega$ & Facility cost, (€/day) & 46.00 \\
\hline$S_{C F}$ & Subsidy to CF operator (€/day) & 0 \\
\hline$\theta_{C F}$ & Fare per parcel paid by carriers, (€/parcel) & 1.000 \\
\hline$\tau^{C F}$ & Stop time per receiver served by CF operator vehicles in strategy B, (h) & 0.025 \\
\hline$e_{j}^{C F}(v)$ & $\begin{array}{l}\text { Emission factor of pollutant } j \text { corresponding to the CF operator vehicle at } \\
\text { cruising speed } v \text { (amount of pollutant } j \text { /unit of distance) }\end{array}$ & 0 \\
\hline \multicolumn{3}{|c|}{ 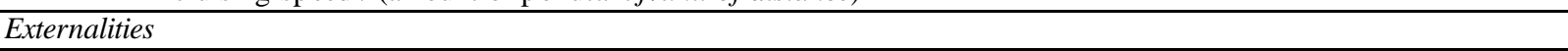 } \\
\hline & $\begin{array}{r}\text { Monetary cost of the impact of an amount of pollutant } j \text { on the society and } \\
\text { the environment }\end{array}$ & \\
\hline & Monetary CO2 Weighting factor $(€ / \mathrm{kg})^{* 3}$ & 0.00768 \\
\hline & Monetary NOxWeighting factor $(€ / \mathrm{kg})^{*_{4}}$ & 6.3 \\
\hline & Monetary PM 2.5 Weighting factor $(€ / \mathrm{kg})^{* 4}$ & 48 \\
\hline
\end{tabular}




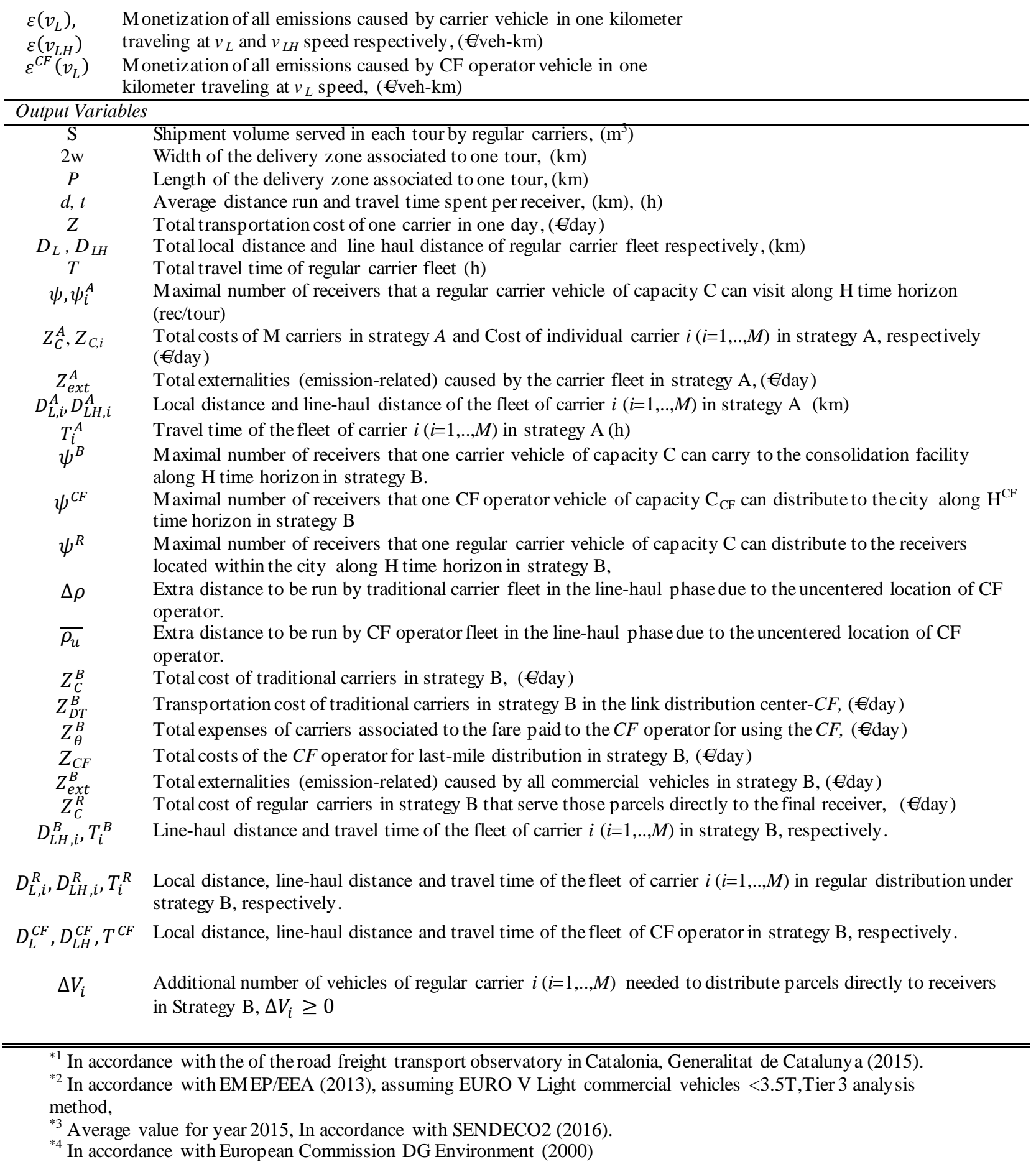

\section{Appendix 2: Lagrangean formulation}

The Lagrangean formulation of the tour design problem is presented here. To estimate the local length of a tour as a function of $w$ and $S$, we extend the contributions of Daganzo (1984a) and divide the rectangle into two bands each of width $w$. Then, each route visits points in non-decreasing coordinate $x$ along the length of the rectangle in the inbound direction and decreasing $x$ in the outbound direction (See Figure 2). If points are randomly distributed over space, one can evaluate the expected total distance. As distances are computed in $\mathrm{L}_{1}$ metric, we split the total distance into the traverse and the longitudinal components that will be calculated independently.

Equation (A.1) determines the objective function to be minimized for a single carrier. The decision variables are the dimension of the vehicle zone delivery partition $(w)$ and the vehicle volume load $(S)$. 


$$
\begin{gathered}
\min _{w, S} N\left[\left(c_{d}+\frac{c_{t}}{v_{L H}}\right) \frac{2 \rho E(y)}{S}+\left(c_{d}+\frac{c_{t}}{v_{L}}\right) \frac{1}{w \delta}+\left(c_{d}+\frac{c_{t}}{v_{L}}\right) \frac{w}{3}+\left(c_{d}+\frac{c_{t}}{v_{L}}\right) \frac{\beta E(y)}{S}+\tau c_{t}\right] \\
S \leq C \\
\frac{2 \rho}{v_{L H}}+\frac{\beta}{v_{L}}+\frac{S}{w \delta v_{L} E(y)}+\frac{w S}{3 v_{L} E(y)}+\frac{\tau S}{E(y)} \leq H \\
w, S \geq 0 \text { continuous }
\end{gathered}
$$

Inequality constraints (A.2)-(A.3) capture the limitations on the capacity of the vehicles, $C$, and the available time horizon, $H$, to visit all receivers. Constraint (A.4) states the non-negative nature of the decision variables.

The constrained minimization problem stated in Equations (A.1)-(A.4) can be solved by means of Lagrange relaxation technique. If we reformulate the constraints (A.2)-(A.3) to be equalities in the form of $g_{1}(w, S)=C-S, g_{2}(w, S)=H-\left(\frac{2 \rho}{v_{L H}}+\frac{S}{w \delta v_{L} E(y)}+\frac{w S}{3 v_{L} E(y)}+\frac{\beta}{v_{L}}+\frac{\tau S}{E(y)}\right)$ respectively, and define the Lagrange multipliers $\lambda_{1}, \lambda_{2}$; the corresponding relaxed problem is defined in Equations (A.5)-(A.10). Equation (A.5) is the Lagrange function and Equations (A.6)-(A.10) plus (A.2)-(A.4) are optimality conditions, where $\pi=\left(c_{d}+\frac{c_{t}}{v_{L}}\right)$ and $\varepsilon=\left(c_{d}+\frac{c_{t}}{v_{L H}}\right)$.

$$
\begin{gathered}
\mathcal{L}\left(w, S, \lambda_{1}, \lambda_{2}\right)=N\left[\frac{2 \rho E(y) \varepsilon}{S}+\frac{\pi}{w \delta}+\frac{w \pi}{3}+\frac{\beta E(y) \pi}{S}+c_{t} \tau\right]-\lambda_{1} g_{1}(w, S)-\lambda_{2} g_{2}(w, S) \\
\frac{\partial \mathcal{L}}{\partial w}=N \pi\left(\frac{1}{3}-\frac{1}{\delta w^{2}}\right)+\lambda_{2} \frac{S}{E(y) v_{L}}\left(\frac{1}{3}-\frac{1}{\delta w^{2}}\right)=0 \\
\frac{\partial \mathcal{L}}{\partial S}=-\frac{2 N \varepsilon \rho E(y)}{S^{2}}-\frac{\beta N \pi E(y)}{S^{2}}+\lambda_{1}+\lambda_{2}\left(\frac{w}{3 E(y) v_{L}}+\frac{1}{\delta w^{2} E(y) v_{L}}+\frac{\tau}{E(y)}\right)=0 \\
\lambda_{2}\left(H-\left(\frac{2 \rho}{v_{L H}}+\frac{\beta}{v_{L}}+\frac{\lambda_{1}(S-C)=0}{w \delta v_{L} E(y)}+\frac{w S}{3 v_{L} E(y)}+\frac{S \tau}{E(y)}\right)\right)=0 \\
\lambda_{1} \geq 0 ; \lambda_{2} \geq 0
\end{gathered}
$$

In this problem, there are two complementarity conditions (Equations 10-11) regarding the former constraints. They generate four cases to check the optimal values of the four variables involved $w, S, \lambda_{1}$, $\lambda_{2}$, satisfying Constraints (A.2)-(A.4), and (A.10): $\left(\lambda_{1}=0\right.$ and $g_{2}=0 ; \lambda_{2}=0$ and $g_{1}=0 ; \lambda_{1}=$ 0 and $\lambda_{2}=0 ; g_{2}=0$ and $\left.g_{1}=0\right)$. However, only the two first cases are feasible, providing the following optimal vector of optimal results: $\left(w, S, \lambda_{1}, \lambda_{2}\right)_{1}^{*}=$ $\left(\left(\frac{3}{\delta}\right)^{1 / 2}, \frac{H-\frac{2 \rho}{v_{L H}}-\beta / v_{L}}{\frac{2}{\sqrt{3} E(y) v_{L} \delta^{1 / 2}}+\frac{\tau}{E(y)}}, 0, \frac{(2 \rho \varepsilon+\beta \pi)^{2}(y)_{N}}{S^{2}\left(\frac{2}{\sqrt{3} v_{L} \delta^{1 / 2}}+\tau\right)}\right),\left(w, S, \lambda_{1}, \lambda_{2}\right)_{2}^{*}=\left(\left(\frac{3}{\delta}\right)^{1 / 2}, C, \frac{(2 \rho \varepsilon+\beta \pi)_{E}(y)_{N}}{C^{2}}, 0\right)$. Note that the values of the decision variables $(w, S)$ and the Lagrange multipliers are always positive for both optimal solutions. There is one exception in the value of $S$ in the first optimal solution: when the travel time in the line-haul phase of one area $\left(2 \rho / v_{L H}\right)$ plus the time needed for detouring $\left(\beta / v_{L}\right)$ is greater than the available time horizon $(H)$. In this case, there is not a feasible solution since there is not enough time to perform the local distribution. Therefore, in the estimation of cost incurred by carrier, the band width will be equal to $w=\left(\frac{3}{\delta}\right)^{1 / 2}$ whereas the number of receivers visited in each route will be $\psi=\frac{S}{[E(y)]}=$ $\min \left\{\frac{C}{[E(y)]} ; \frac{H-2 \rho / v_{L H}-\beta / v_{L}}{\frac{2}{\sqrt{3 \delta v_{L}}}+\tau}\right\}$. The latter decision variable will vary depending on whether Constraint (A.3) is tight or not. In this modeling approach, the required number of carrier routes entering the city has been estimated by the real term $N / \psi$ although this is an integer value in reality. This mathematical approximation should be allowed when $N \gg>\psi$. 


\section{Appendix 3: Factor diagrams}

The estimations of the factors to be used in Equations (26), (28), and (30) are provided in the following Figures.

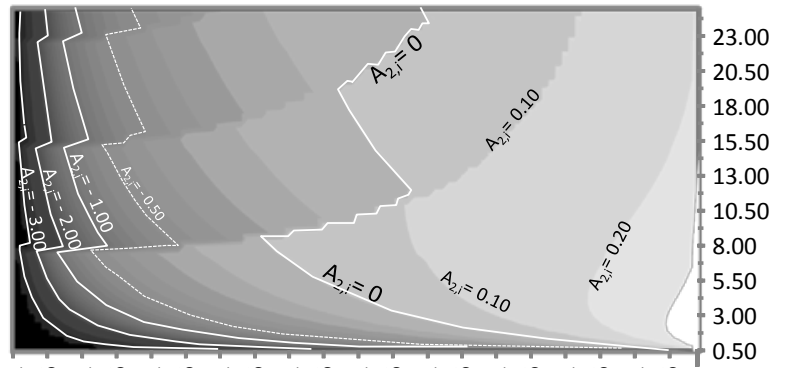

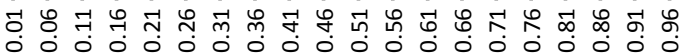

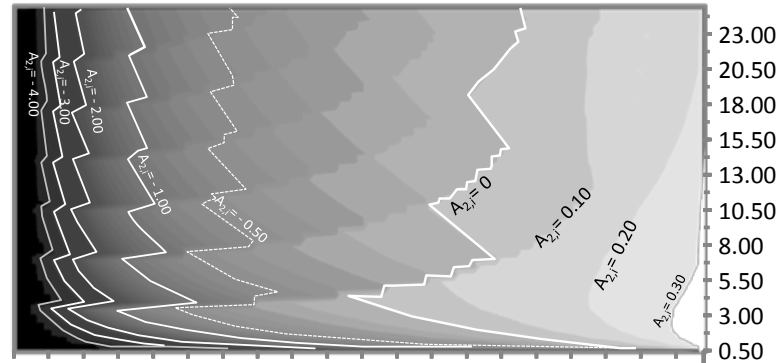

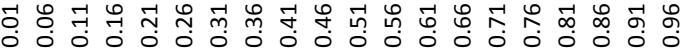

$$
F\left(u_{\max }\right)
$$

$$
F\left(u_{\max }\right)
$$

(a)

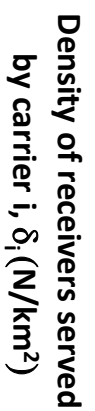

(b)

Figure A1. Estimation of parameter $A_{2, i}$ when a) $C=9 m^{3}$ and b) $C=4.2 m^{3}$

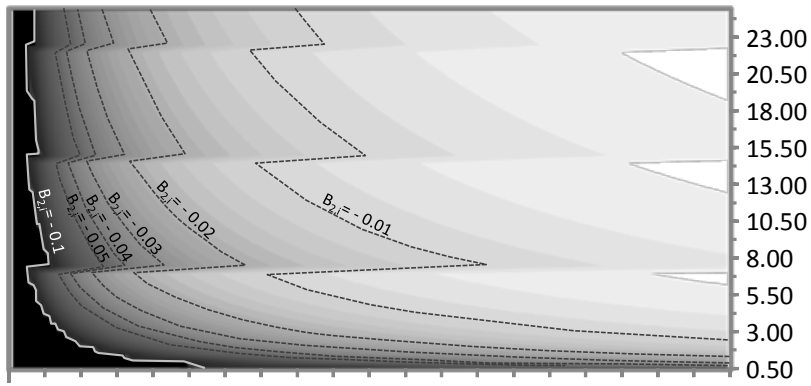

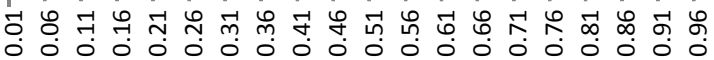

$$
F\left(u_{\max }\right)
$$

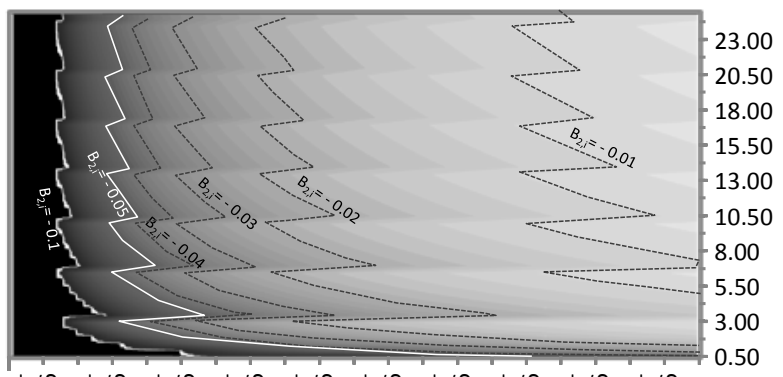

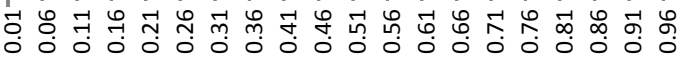

$F\left(u_{\text {max }}\right)$

Figure A2. Estimation of parameter $B_{2, i}$ when a) $C=9 m^{3}$ and b) $C=4.2 m^{3}$

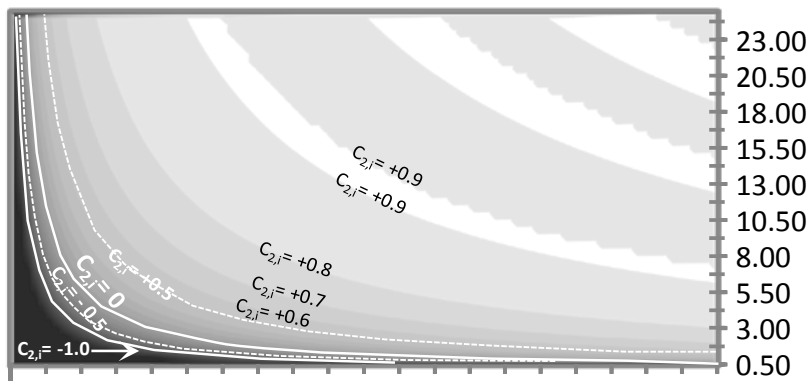

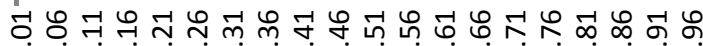

$$
F\left(u_{\max }\right)
$$

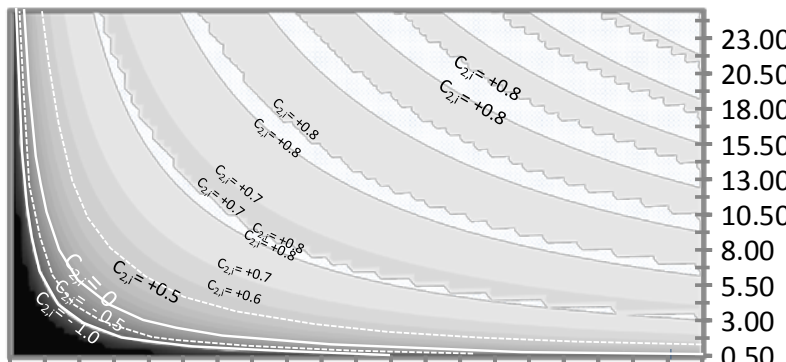

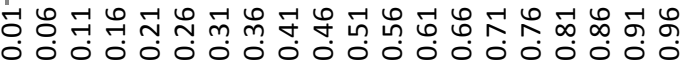

$$
F\left(u_{\max }\right)
$$

Figure A3. Estimation of parameter $C_{2, i}$ when a) $C=9 \mathrm{~m}^{3}$ and b) $C=4.2 \mathrm{~m}^{3}$ 


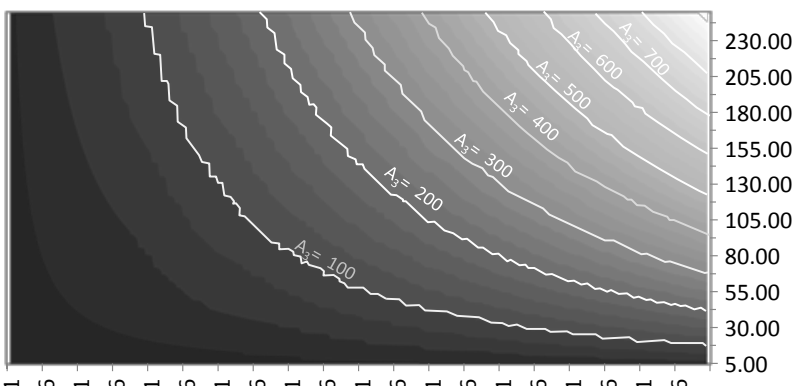

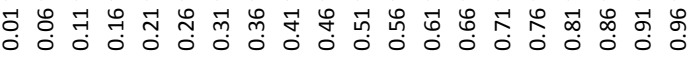

$$
F\left(u_{\max }\right)
$$

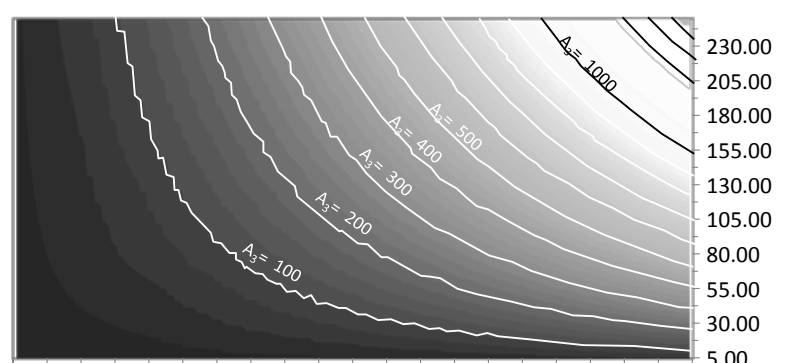

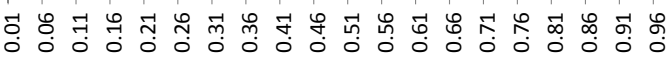

$\mathbf{F}\left(\mathbf{u}_{\max }\right)$

(b)

Figure A4. Estimation of parameter $A_{3}$ when a) $C_{C F}=2.16 m^{3}$ and b) $C_{C F}=1.2 m^{3}$

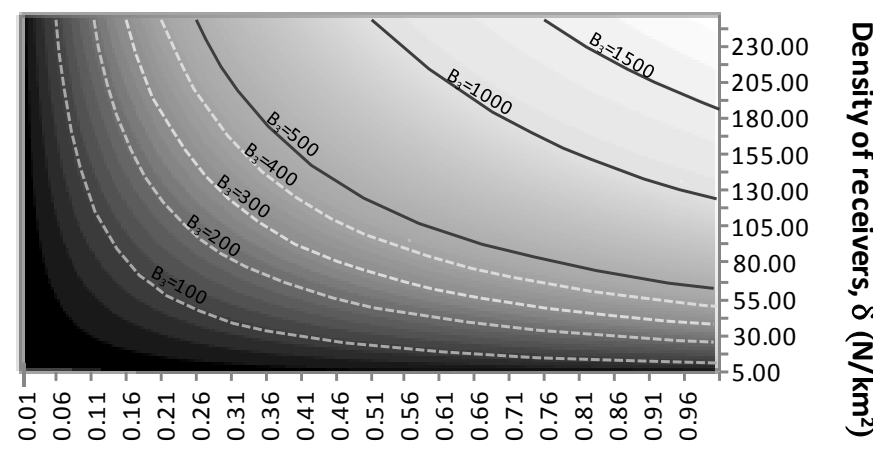

$\mathbf{F}\left(\mathbf{u}_{\max }\right)$

Figure A5. Estimation of parameter $\mathrm{B}_{3}$ 